\title{
Development of Doppler Global Velocimetry as a Flow Diagnostics Tool
}

James F. Meyers

NASA Langley Research Center

Hampton, Virginia

Measurements in Fluids and

Combustion Systems

Special Issue

Measurement Science and Technology

vol. 6, nu. 6, pp. 769-783

June 1995 



\title{
Development of Doppler Global Velocimetry as a Flow Diagnostics Tool
}

\author{
James F. Meyers \\ Mail Stop 493 \\ National Aeronautics and Space Administration \\ Langley Research Center \\ Hampton, Virginia 23681-0001 \\ Development of Doppler Global Velocimetry
}

\begin{abstract}
The development of Doppler global velocimetry is described from its inception to its use as a flow diagnostics tool. Its evolution is traced from an elementary one-component laboratory prototype, to a full three-component configuration operating in a wind tunnel at focal distances exceeding $15 \mathrm{~m}$. As part of the developmental process, several wind tunnel flow field investigations were conducted. These included supersonic flow measurements about an oblique shock, subsonic and supersonic measurements of the vortex flow above a delta wing, and three-component measurements of a high-speed jet.
\end{abstract}

\section{Introduction}

In 1991, Komine et al (1991) presented a new approach to global velocity measurements. This technique-Doppler Global Velocimetry - had the potential to provide global, three-component, velocity measurements at video frame rates using standard video cameras. If this technology can be developed into a wind tunnel flow measurement system, experimental data could be obtained with grid spacings comparable to CFD solutions, with near real-time acquisition and processing. Toward this end, NASA Langley Research Center has been conducting a research program to develop this concept into a flow field diagnostics tool. The following is a synopsis of that program, including example results from several flow field investigations conducted in its support.

\section{Basic Principles}

In the manner of the original laser velocimeter developed by Yeh and Cummins (1964), Doppler Global Velocimetry (DGV) determines the velocity of micron sized particles embedded in the flow by measuring the Doppler shift frequency of the scattered light as the particles pass through a laser beam. As depicted in figure 1, scattered light collected 
by a detector located along the direction ô, from particles passing through a laser beam propagating in direction $\hat{1}$, is Doppler shifted based on a velocity in the direction $(\hat{o}-\hat{1})$. This relationship is expressed by:

$$
\Delta v=\frac{v_{o}(\hat{o}-\hat{1}) \cdot V}{c}
$$

where $\Delta v$ is the Doppler shifted frequency, $v_{0}$ is the laser frequency, $\mathbf{V}$ is the particle velocity, and $c$ is the speed of light.

The principle differences between the reference-beam laser velocimeter, DGV, and the other methods that measure the Doppler shift of scattered laser light are found in the receiver optical system. The reference-beam laser velocimeter, and arguably the fringe-type configuration, measure the Doppler shift using a square-law detector (photodiode or photomultiplier) to heterodyne the collected scattered light with a portion of the reference beam (or scattered light from the second laser beam as derived by Meyers (1971)). Jackson and Paul (1970) used a Fabry-Perot interferometer to simultaneously measure the optical frequencies of the laser output and the scattered light. The difference between the two spectral peaks was the Doppler shift frequency. Smeets (1984) used a phase stabilized Michelson interferometer as a high resolution spectrometer to yield a signal proportional to the Doppler shift frequency. Komine et al (1990), Miles et al (1992), and Elliottet al (1993) used the side of an absorption line in Iodine vapor as an optical frequency discriminator to measure the Doppler shift frequency. Although these efforts formed the basis of DGV, later work by Hoffenberg and Sullivan (1993) and Roehle and Schodl (1994) developed point measurement systems based on this technology. Kremer et al (1993) developed a long range system based on Faraday magneto-optic atomic line filters. This technique uses the polarization characteristics of a Faraday cell as an optical frequency discriminator.

Of these techniques, the Michelson interferometer, the Iodine vapor cell, and the Faraday cell methods have the potential of being used as the basis of a global velocity measurement system. Seiler and Srulijes (1986) developed a global measurement system based on a Michelson interferometer to measure the instantaneous velocity field in a shock tube. Their Doppler pictures consisted of interferometric fringes of constant velocity. While the Iodine vapor cell is used in DGV, a Faraday cell could also be used following the necessary modifications. To date, this approach has not been implemented. 
Iodine vapor has numerous absorption lines in the green part of the visible spectrum. Several lines lie within the optical tuning range of a single-frequency, frequency-doubled Nd:YAG laser, and one lies within the tuning range of an Argon ion laser set to $514.5 \mathrm{~nm}$ (Komine et al (1994)). With an Iodine vapor cell set to $45^{\circ} \mathrm{C}$, the absorption line at $514.5 \mathrm{~nm}$ was mapped, figure 2 . If the laser output frequency was tuned to a point midway along the edge of the absorption line, figure 2, collected scattered light from a stationary object or cloud of particles would be attenuated by 50 percent as it passed through the vapor. If the object or particle cloud were moving, the attenuation through the vapor increased (or decreased, depending on the direction of movement) by an amount proportional to the scattered light Doppler shift frequency induced by the object's motion, equation (1). Thus, placing an Iodine vapor cell in front of the detector in figure 1, the level of the detector output would be proportional to the velocity of an object or particle cloud passing through the laser beam. Unfortunately, the level would also be proportional to the total amount of light scattered. Thus, influences from particle number density, particle size distribution, and intensity variations across the laser beam must be removed. A beam splitter was placed ahead of the Iodine cell to direct a portion of the collected light toward a second detector. This detector provided the voltage level needed to produce a normalized signal whose amplitude was influenced only by the Iodine vapor.

A DGV optical system was created by expanding the input laser beam into a light sheet, and replacing the two detectors by Charge Coupled Device (CCD) video cameras, figures 3 and 4 . Since each camera pixel becomes, in effect, a single detector, a system using 512-x 512-pixel cameras would be equivalent to 262,144 fringe-type laser velocimeters. A three-component DGV system can be constructed by adding two additional receivers at different angles to the light sheet, i.e., three different output vectors, $\hat{o}_{a}$, $\hat{o}_{b}$, and $\hat{o}_{c}$, in equation (1).

\section{The Development of the DGV Concept}

The invention of Doppler Global Velocimetry by Komine (1990) suggested that three-component global velocity measurements, with grid spacings equivalent to those used in CFD, were possible with a simple optical system. The potential of the concept, along with its simplicity, made it an excellent candidate for development in to a flow diagnostics instrument. In 1990 the NASA Langley Research Center began an accelerated program to develop this technology for wind tunnel and flight applications. 


\subsection{The Laboratory Development}

The desire by NASA researchers to accelerate the development process of DGV prompted a dual-effort approach. Komine and his team at the Northrop Research and Technology Center were contracted to determine the feasibility of using a pulsed, frequency-doubled Nd:YAG laser as the DGV laser source under contract to Langley (Komine et al (1994)). The Northrop program was to determine the necessary laser characteristics for DGV operation, and the potential of a pulsed system for flight applications. At the same time, an in-house effort would construct an Argon-ion laser based system, duplicating the laboratory configuration constructed by Komine (1990). This undertaking would provide an in-house understanding of the technique, and an opportunity for testing the technology in a wind tunnel environment.

\subsubsection{Investigation of the Nd:YAG laser based DGV system}

The effort at Northrop (Komine et al (1994)) found that a DGV optical system could be constructed using a frequency-doubled Nd:YAG laser. The laser must be injection locked to single-frequency operation, with the capability of tuning the output laser frequency to match an absorption line of Iodine vapor. The injection seed laser output frequency was controlled through an active feedback loop, which utilized a second Iodine cell to monitor the Nd:YAG output. A piezoelectric drive was used to optimize the Nd:YAG laser cavity length for the chosen optical frequency. The laser mode structure was monitored using a 1 -meter long vacuum spaced etalon. The resulting ring pattern was imaged on a CCD video camera for continuous monitoring of the detailed structure.

Even with the efforts to insure single-frequency operation of the $\mathrm{Nd}$ :YAG laser, the optical bandwidth was approximately $100 \mathrm{MHz}$, an order of magnitude greater than the bandwidth of an Argon ion laser. To determine if measurement accuracy could be maintained even with the increased bandwidth, a comparative investigation with a pitot probe was conducted in a subsonic jet flow. Example results, shown in figure 5, indicate that a pulsed, frequency-doubled Nd:YAG laser could be used in DGV. Further, by synchronizing the electronic shutters of CCD video cameras with the laser pulse, background sky light can be reduced to negligible levels, allowing DGV measurements to be made. Thus, a DGV optical system designed around an Nd:YAG laser would be feasible for flight applications. 


\subsubsection{Developing a Laboratory DGV System at NASA}

The development program began at Langley (Meyers and Komine (1991)) with the construction of a prototype one-component DGV optical system. A custom electronics circuit was constructed to acquire the two United States standard RS-170 video signals from the monochrome signal and reference cameras respectively. The circuit would strip the synchronization pulse, and divide the signal camera output by the reference camera output with an analog divider. The synchronization pulse was returned to the normalized output, forming a standard RS-170 signal that was now proportional to velocity.

Although each video camera was placed on optical mounts with horizontal, vertical, pan, and tilt motion capabilities, exact alignment of the two cameras was not possible. The problem was traced to distortions in the optics. Thus, the simple analog divider circuit would not provide the necessary measurement accuracy, although it did provide a real-time estimate of the velocity field. A digital frame grabber was needed to allow the manipulation of the acquired image before normalization to remove any optical distortions. Since standard frame grabbers of the time could not be synchronized to acquire simultaneous images from two cameras, a custom dual frame grabber was constructed. Standard image warping software was also found lacking. The minor, though complex, image distortions could not be corrected by standard warping algorithms that adjust the entire image. Custom software based on piecewise bilinear warping proved to be the answer (Meyers (1992)). Warping the two images before normalization yielded the subpixel overlay accuracy needed for accurate measurements.

Following successful measurements of a rotating wheel, the system was installed about the NASA Basic Aerodynamic Research Tunnel, BART, figure 4. The object of this first wind tunnel test was the measurement of the vortical flow above a 75-degree delta wing. This flow field was selected because the velocity structure was similar to the spinning wheel, and comparative three-component laser velocimetry data were available (Meyers and Hepner (1988)). The investigation proved to be an examination of the DGV system, and not the flow field. The following modifications were made to the system in response to the problems uncovered by the study. The cylindrical lens used to create the light sheet was replaced with a high-speed galvanometer scanner. The Gaussian intensity profile of the lens generated light sheet, coupled with variations in particle number density, resulted in scattered light levels that exceeded the dynamic range of the camera. Saturation levels occurred at the center of the light sheet, and insufficient signal strength was found at the edges. By sweeping the beam, a flatter light sheet 
intensity profile was obtained than the Gaussian profile produced with the cylindrical lens. The scanner was operated at $130 \mathrm{~Hz}$ to insure illumination of each point within the light sheet at least twice during the $16.7 \mathrm{msec}$ acquisition time for each camera field. In effect, the $16.7 \mathrm{msec}$ light integration time for each camera pixel was reduced to the time for two beam passage occurrences of approximately $0.01 \mathrm{msec}$ each.

Polarization effects were also found to cause problems. Mie (1908) found that illumination of small particles with linearly polarized light produced scattering light intensities that varied greatly as a function of scattering angle. These variations could result in levels that exceeded the dynamic range of the cameras, especially the lower limit. A quarterwave plate was placed in the linearly polarized laser beam to circularize the beam polarization. This reduced the scattered light intensity variations to an acceptable level. A second effect of polarization was the variation in the transmission/reflection ratio by the beamsplitter in the receiver. The ratio of signal level with reference level would vary in a velocity independent manner if the polarization of the scattered light varied because of changing characteristics in the particle field. The polarization dependency was lowered by reducing the angle of the beamsplitter from 45 degrees to the optical axis to 20 degrees.

The majority of the image distortions were traced to the scattered light collecting zoom lens and its associated relay lens. Removing these lenses to convert the receiver to the boresight configuration, used by Komine (1991), improved the image quality. An added benefit of the increased transmission of collected scattered light was an increase in signal-to-noise ratio. Other image distortions were found to originate from changes in the particle cloud structure between the acquisition of the two interlaced camera fields comprising each image frame. Since the even lines acquire photons $16.7 \mathrm{msec}$ before the odd lines, the particle cloud shape and position, along with the velocity structures, were different in the two fields. By separating the even lines from the odd lines, and processing each field separately, image quality was improved.

With the above modifications in place, the investigation of the vortical flow above the 75-degree delta wing began. The results of the investigation were reported by Meyers et al (1992). An example velocity image of the nearly cross flow component ( $0.32 \mathrm{i}-0.95 \mathrm{j})$ at 20.5 -degrees angle-of-attack is shown in figure 6. Comparable three-component, fringe-type laser velocimeter measurements, obtained earlier by Meyers and Hepner (1988), were resolved along the same velocity vector direction, and are presented in figure 7 . While the laser velocimeter measurements exhibit the expected velocity contours for stable counter 
rotating vortices, only the left vortex was found to be stable in the DGV measurements. The right vortex exhibited the characteristics found from a solid body of revolution, such as the rotating wheel. These data may represent the transition from a stable vortex to a burst condition. This hypothesis was supported by the flow visualization results shown in figure 8. Although the investigation was carefully set to repeat the earlier laser velocimeter test, the data indicate differences existed between the two studies.

The delta wing was then set to 40-degrees angle of attack, and data images acquired of the burst vortical flow. The average of 30 frames (60 fields) of DGV data show structures in the cross flow component very similar to those found in comparable laser velocimetry data, figures 9 and 10 respectively. The laser velocimetry measurements of the burst vortical flow found standard deviations exceeding 30 percent of the local mean velocity (Meyers and Hepner (1988)). If the large standard deviations were due to random turbulence, random variations in velocity would obscure the flow structure in individual images comprising the average structures shown in figure 9 . Instead, each of the four individual field images shown in figure 11 have distinctive, though different, flow structures. This may represent the first data indicating that burst vortices maintain velocity structures, though they vary greatly with time.

The delta wing was replaced by an F/A-18 model to determine if the vortical flow structures above the model at high angles of attack were similar to those above the delta wing. The receiver optics were moved to an overhead position to avoid blockage by the vertical stabilizers, figure 12. An example of the results for 25-degrees angle-of-attack, reported by Lee et al (1993), is shown in figure 13. The data indicate that the primary vortices from the leading edge extensions have similar structures to the burst vortical flow above the delta wing.

\subsection{Additional System Modifications}

Although the investigation in the BART produced results that compared favorably with previous laser velocimetry data, evidence of additional problems was found. The velocity measurements appeared to slowly drift during data acquisition, indicating that the laser frequency was changing with time. Additionally, large increases in measured velocity occurred when the particle density was low, notably at the edges of the smoke plume.

Subsequent laboratory investigations found that the laser frequency did drift with time, and occasionally would shift longitudinal modes, 
even though the etalon was temperature stabilized. The drift could be removed by constructing an active feedback control system that adjusts the laser cavity length to compensate for changes within the laser. This approach was used by Roehle and Schodl (1994) to yield an uncertainty of $\pm 2 \mathrm{~m} \mathrm{~s}^{-1}$. Komine et al (1994) also used this approach to stabilize their $\mathrm{Nd}$ :YAG laser used in the pulsed DGV optical system. Instead of removing the drift, the laboratory investigations indicated that monitoring the laser output frequency using a second Iodine vapor cell would yield similar measurement uncertainties. The longitudinal mode structure was also visually monitored using a standard optical spectrum analyzer. Adjustment of the laser to regain single mode operation took place if any deviation from single mode was seen. During data acquisition, the transfer function of the second or reference Iodine vapor cell was sampled for each measurement image acquired. The measured reference level yielded the optical frequency bias equated to still air in the corresponding data image. This frequency bias was subtracted from the measurement image values to compensate for the laser drift.

Further laboratory investigations were conducted to determine the characteristics of the CCD cameras in an effort to find the cause for the large velocity increases at the edge of the smoke plume. The study revealed two principle sources of random noise, variations in pixel-topixel sensitivity and charge transfer noise. Their effects were then minimized using standard image processing techniques. Variations in pixel sensitivity could be removed by calibrating the gain for each pixel, then using the calibrations to flatten the camera response. While charge transfer noise, (noise imposed on a pixel charge packet as it passed from the acquiring pixel to the camera output port), could not be removed, its effect could be lessened by low pass filtering the image. The standard deviation of the resulting noise was less than $\pm 2 \mathrm{~m} \mathrm{~s}^{-1}$ based on DGV measurements in an empty wind tunnel.

These noise sources, however, did not account for the large velocity increases at the edge of the smoke plume. Subsequent analysis of the signal and reference images revealed that minor misalignments occurred between the two cameras. These misalignments caused signal levels obtained from scattered light originating from one part of the light sheet being normalized by scattered light levels obtained from another part. These errors were small when the particle cloud was nearly uniform, but when a steep gradient was present, such as the edge of the cloud, the variations become very large. Spatially cross correlating the signal and reference images provided the misalignment offset. Shifting the signal image to negate the misalignment before normalization greatly reduced the errors at the edges of the particle 
cloud. The remaining errors were simply removed by trimming each edge by four pixel locations.

\subsection{Wind Tunnel Testing}

The next step in the development of Doppler Global Velocimetry was the determination of the maximum limits in working focal distance and measurable velocity. The investigations of a jet flow in the Langley 14-x 22 -foot Subsonic Tunnel, and the flow about an oblique shock in the Langley Unitary Plan Wind Tunnel were conducted to aid in this effort.

The investigation in the 14-x 22 -foot Subsonic Tunnel was to determine the flow about a powered model engine mounted to a semispan model of a large transport aircraft. The primary concern was whether the reverse flow from the engine compressor blades would interact with free stream to then flow over the wing and be reingested by the aft engine intakes. A mosaic of three overlapping 0.5-x 0.5-meter measurement planes were needed to map the flow field. The velocity field for the component $-0.32 \mathrm{i}$ $-0.67 \mathrm{j}+0.68 \mathrm{k}$ (upstream, above airfoil, and spanwise, respectively) is shown in figure 14. The results indicated that the reverse flow did interact with free stream to flow over the wing, but was not reingested. The only difficulty with the optical system was the expected decrease in collected scattered light.

The investigation conducted in the Unitary Plan Wind Tunnel consisted of several elements to gain insight into the characteristics of DGV when used for supersonic flow measurements (Meyers (1993)). The first element was the measurement of the flow in the empty tunnel test section to determine if water condensation could be used as the seed material. An earlier investigation using a laser transit anemometer, capable of detecting 0.2 micron diameter particles, was unable to resolve the condensation particles. Thus the condensation particles were expected to be less than 0.2 microns in diameter. The DGV test proved successful with sufficient scattered light to yield the expected flat velocity profile. A flat plate was then installed in the test section and inclined to -15 degrees to obtain a flow with an oblique shock. The measured velocity profile across the shock could be used, along with predictions of the particle dynamics developed by Walsh (1976), to estimate the size of the condensation particles. The resulting velocity map of the vertical component, showing a well-defined oblique shock for a free stream Mach number of 2.5, is presented in figure 15. The sharpness of the measured shock is more easily viewed by presenting the velocities along a single row of pixels in the standard X-Y format, figure 16 . While the velocity lag was only $2 \mathrm{~mm}$, it was greater than expected for the small condensation particles. This inconsistency was 
traced to a characteristic of imaging optics and CCD cameras, the Modulation Transfer Function (MTF). For example, a photograph taken at an f16 lens opening produces a sharper image than the same photograph taken at an $\mathrm{f} 2$ lens opening. The sharpness factor or spatial frequency limit is the MTF of the lens system. Likewise, the MTF of a CCD video camera is a measure of the image sharpness. The lens system MTF is related to its optical characteristics. However, the camera MTF is a measure of the electronic charge leakage between adjacent pixels. The response of the camera system to a spatial step change in intensity is shown in figure 16, and represents the combined MTF. The close match between the MTF and DGV measurements indicated that the apparent particle lag was caused by the limits of the instrumentation and not particle dynamics. The MTF also established the spatial bandwidth of the data images. Thus the value of the MTF could be chosen as the bandwidth for the spatial low pass filter used to reduce the charge transfer noise, since frequencies higher than the MTF could only originate from electronic noise.

The final investigation in the Unitary Plan Wind Tunnel was the measurement of the vortical flow above a 75 -degree delta wing at various angles of attack. Whereas only the vertical component of velocity was measured in the previous elements, multiple components of velocity were desired to fully describe the vortical flow. Unfortunately, the expected Doppler shift for the streamwise component exceeded the measurable range. The solution was to locate the laser frequency along the left edge of the Iodine absorption line, figure 2, and measure the Doppler shifted frequency on the right edge. This approach was used to make measurements up to the wind tunnel limit of Mach 4.6.

The measure of the vortical flow presented the additional opportunity of investigating a complicated flow containing uniform particle number densities. Flow visualization of this flow, figure 17, showed three distinct scattered light intensity levels: free stream, behind the cross flow shock, and the vortex. The velocity image, figure 18, did not yield the expected change in velocity at the intensity boundaries. This anomaly was later traced to the inclusion of background light in both the signal and reference images, resulting in measurement errors. The background light was traced to camera dark current, reflected laser radiation from surfaces, and ambient lights. The influence of ambient light was reduced by placing a green photographic filter in front of each camera. The remaining background light could be measured by acquiring images under normal conditions without particles in the flow. These background images were then subtracted from the matching data images prior to normalization. 
The uniform particle number density also allowed the identification of transmission differences between the signal and reference optical paths in the receiver. These differences resulted in nonvelocity related variations of the measurement ratios. Transmission variations could be caused by polarization effects on the transmission characteristics of the beamsplitter, Iodine plating on the vapor cell windows, dirt on the tunnel windows, etc. The transmission profile could be obtained by performing a normal data acquisition with the laser frequency adjusted to be outside the absorption line. The resulting normalized image was then inverted and used as a multiplying transfer function to negate the differences in transmission between the signal and reference optical paths.

\section{The Three-Component DGV Optical System}

The true potential of Doppler Global Velocimetry could begin to be investigated with the addition of two receiver optical systems to obtain full three-component measurement capability. The laboratory development of the system included the necessary modifications to the data acquisition hardware and software, data processing software, and measurements of a rotating wheel. Once the system was developed, a second system was designed for use at the NASA Ames Research Center. Once constructed, the Ames system was used in the 40-x 80-foot Wind Tunnel to measure the flow from a high-speed jet as a demonstration of three-component DGV.

\subsection{Laboratory Development of Three-Component DGV}

Since the additional two receiver optical systems were constructed identical to the original, the extension to a three-component optical system was straight forward. Likewise, the data acquisition electronics were a duplicate of the single component system, i.e., six synchronized frame grabbers versus two. The data processing software required modification to acquire three nonorthogonal velocity component images, then resolve them into standard Cartesian coordinates.

The three-component DGV optical system was placed about a rotating wheel, with a receiver set on the left, right, and above with a 30 -degree angle to the plane of the wheel. The wheel was illuminated with a cone of laser light propagating in the horizontal plane, and inclined by 45 degrees to the wheel. The view from each receiver of the calibration card is shown in figure 19. The perspective and optical distortions were removed using the normal piecewise bilinear warping software, except the number of rows and columns extending from the central dot were 
matched in each image. The resulting warped views are shown in figure 19. Thus, all six camera images had the proper overlay to determine the standard $u, v$, and $w$ velocity components. The standard components were calculated for each pixel because of the minor differences in the measured velocity vectors for each location within the light sheet.

The wheel was spun and the velocity data acquired. The measured and converted velocity images are shown in figure 20. As expected, the $u$ component was nominally $0 \mathrm{~m} \mathrm{~s}^{-1}$, and the $v$ and $w$ components had the appropriate profiles for a solid body of revolution, with the proper 90 degree phase difference.

\subsection{Wind Tunnel Demonstration of Three-Component DGV}

The first wind tunnel application of three-component Doppler Global Velocimetry served as a demonstration of the Langley-designed system for the 40-x 80-foot Wind Tunnel at the NASA Ames Research Center (Meyers (1994)). The system orientation for the measurement of the high-speed jet flow from an HSCT engine model is shown in figure 21. The study provided resolved $u, v$, and $w$ velocity components of the $0.22 \mathrm{~m}$ diameter jet, operating at temperatures up to $700^{\circ} \mathrm{C}$ with velocities over $500 \mathrm{~m} \mathrm{~s}^{-1}$. An example image, figure 22, of the streamwise velocity component obtained within a plane normal to the jet axis, 0.25 jet diameters downstream from the exit, clearly shows the velocity deficit caused by the conical central body. The measurement grid capability of the technique, even at focal distances up to $15.5 \mathrm{~m}$, is shown by the X-Y plot of the measured velocities obtained from a single row of pixels with a spatial resolution of $1.25 \mathrm{~mm}$ along the diameter of the jet.

\section{Concluding Remarks}

The development of Doppler Global Velocimetry from an idea to a usable wind tunnel flow diagnostics tool has been presented. A combination of laboratory and wind tunnel investigations have resulted in an instrument that can measure three-component velocities at focal distances greater than $15 \mathrm{~m}$, with a spatial resolution of $1.25 \mathrm{~mm}$. At its current stage of development, the author and others have identified velocity uncertainties of approximately $\pm 2 \mathrm{~m} \mathrm{~s}^{-1}$. This measurement uncertainty was found to be independent of mean velocity. Example velocity measurements obtained from wind tunnel investigations in flows from subsonic to Mach 2.8 were presented. 


\section{References}

Bloom, S. H.; Searcy, P. A.; Choi, K.; Kremer, R.; and Korevaar, E. J.: Helicopter Plume Detection by Using an Ultranarrow-band Noncoherent Laser Doppler Velocimeter. Optics Letters, Vol. 18, No. 3, February 1, 1993.

Elliott, G. S.; Samimy, M.; and Arnette, S. A.: Molecular Filter-Based Diagnostics in High Speed Flows. AIAA 31st Aerospace Sciences Meeting \& Exhibit, Paper 93-0512, Reno, NV, January 11-14, 1993.

Hoffenberg, R.; and Sullivan, J. P.: Filtered Particle Scattering: Laser Velocimetry Using an Iodine Filter. Proceedings of ASME Fluids Engineering Conference, Washington, DC, FED-vol. 161, June 20-24, 1993, pp. 135-138.

Jackson, D. A.; and Paul, D. M.: Measurement of Hypersonic Velocities and Turbulence by Direct Spectral Analysis of Doppler Shifted Laser Light. Physics Letters, vol. 32A, no. 2, June 15, 1970, pp. 77, 78.

Komine, H.: System for Measuring Velocity Field of Fluid Flow Utilizing a Laser-Doppler Spectral Image Converter. United States Patent 4,919,536, April 24, 1990.

Komine, H.; Brosnan, S. J.; Litton, A. B.; and Stappaerts, E. A.: RealTime, Doppler Global Velocimetry. AIAA 29th Aerospace Sciences Meeting, Paper 91-0337, Reno, NV, January 7-10, 1991.

Komine, H.; Brosnan, S. J.; Long, W. H.; and Stappaerts, E. A.: Doppler Global Velocimetry Development of a Flight Research Instrumentation System for Application to Non-Intrusive Measurements of the Flow Field. NASA CR-191490, January 26, 1994.

Kremer, R. M.; Korevaar, E. J.; and Bloom, S. H.: Laser Radar Device. United States Patent 5,267,010, November 30, 1993.

Lee, J. W.; Meyers, J. F.; Cavone, A. A.; and Suzuki, K. E.: Doppler Global Velocimetry Measurements of the Vortical Flow Above an F/A-18. AIAA 31st Aerospace Sciences Meeting \& Exhibit, Paper 93-0414, Reno, NV, January 11-14, 1993.

Meyers, J. F.: Investigation and Calculations of Basic Parameters for the Application of the Laser Doppler Velocimeter. NASA TN D-6125, April 1971. 
Meyers, J. F.; and Hepner, T. E.: Measurement of Leading Edge Vortices from a Delta Wing Using a Three Component Laser Velocimeter. AIAA 15th Aerodynamic Testing Conference, San Diego, CA, Paper 88-2024, May 18-20, 1988.

Meyers, J. F.; and Komine, H.: Doppler Global Velocimetry - A New Way to Look at Velocity. Laser Anemometry: Advances and Applications, 1991, eds. A. Dybbs \& B. Ghorashi, ASME, 1991.

Meyers, J. F.: Doppler Global Velocimetry - The Next Generation? AIAA 17th Aerospace Ground Testing Conference, Paper 92-3897, Nashville, TN, July 6-8, 1992.

Meyers, J. F.; Lee, J. W.; and Cavone, A. A.: Three Component Doppler Global Velocimeter Measurements of the Flow Above a Delta Wing. Sixth International Symposium on Applications of Laser Techniques to Fluid Mechanics, Paper 13.2, Lisbon, Portugal, July 20-23, 1992.

Meyers, J. F.: Looking at Extremes with Laser Velocimetry. Proceedings of the 11th International Invitational Symposium on the Unification of Analytical, Computational, and Experimental Solution Methodologies, pp. 491-516, Danvers, MA, August 18-20, 1993.

Meyers, J. F.: Development of Doppler Global Velocimetry for Wind Tunnel Testing. AIAA 18th Aerospace Ground Testing Conference, Paper 94-2582, Colorado Springs, CO, June 20-23, 1994.

Mie, G.: Optics of Turbid Media. Ann. Phys., vol. 25, no. 3, 1908, pp. 377-445.

Miles, R. B.; Forkey, J. N.; and Lempert, W. R.: Filtered Rayleigh Scattering Measurements in Supersonic/Hypersonic Facilities. AIAA 17th Aerospace Ground Testing Conference, Paper 92-3894, Nashville, TN, July 6-8, 1992.

Roehle, I.; and Schodl, R.: Evaluation of the Accuracy of the Doppler Global Technique. Proceedings of Optical Methods and Data Processing in Heat and Fluid Flow, City University, London, April 14-15, 1994, pp. 155-161.

Seiler, F.; and Srulijes, J.: Doppler-Pictures of Velocity Fields, An Application to Fluid Mechanics. Proceedings of the Third International Symposium on Applications of Laser Anemometry to Fluid Mechanics, Lisbon, Portugal, Paper 19.1, July 7-9, 1986. 
Smeets, G.: Laser Doppler Velocimetry with a Michelson Spectrometer. Laser Anemometry in Fluid Mechanics, Selected Papers from the First International Symposium on Applications of LaserDoppler Anemometry to Fluid Mechanics, R. J. Adrian, D. F. G. Durão, F. Durst, H. Mishina and J. H. Whitelaw, eds., Ladoan-Instituto Superior Técnico, Lisbon, Portugal, 1984, pp. 355-370.

Walsh, M. J.: Influence of Particle Drag Coefficient on Particle Motion in High-Speed Flow With Typical Laser Velocimeter Applications. NASA TN D-8120, 1976.

Yeh, Y.; and Cummins, H. Z.: Localized Fluid Flow Measurements with a He-Ne Laser Spectrometer. Applied Physics Letters, vol. 4, no. 10, pp. 176-178, May 1964.

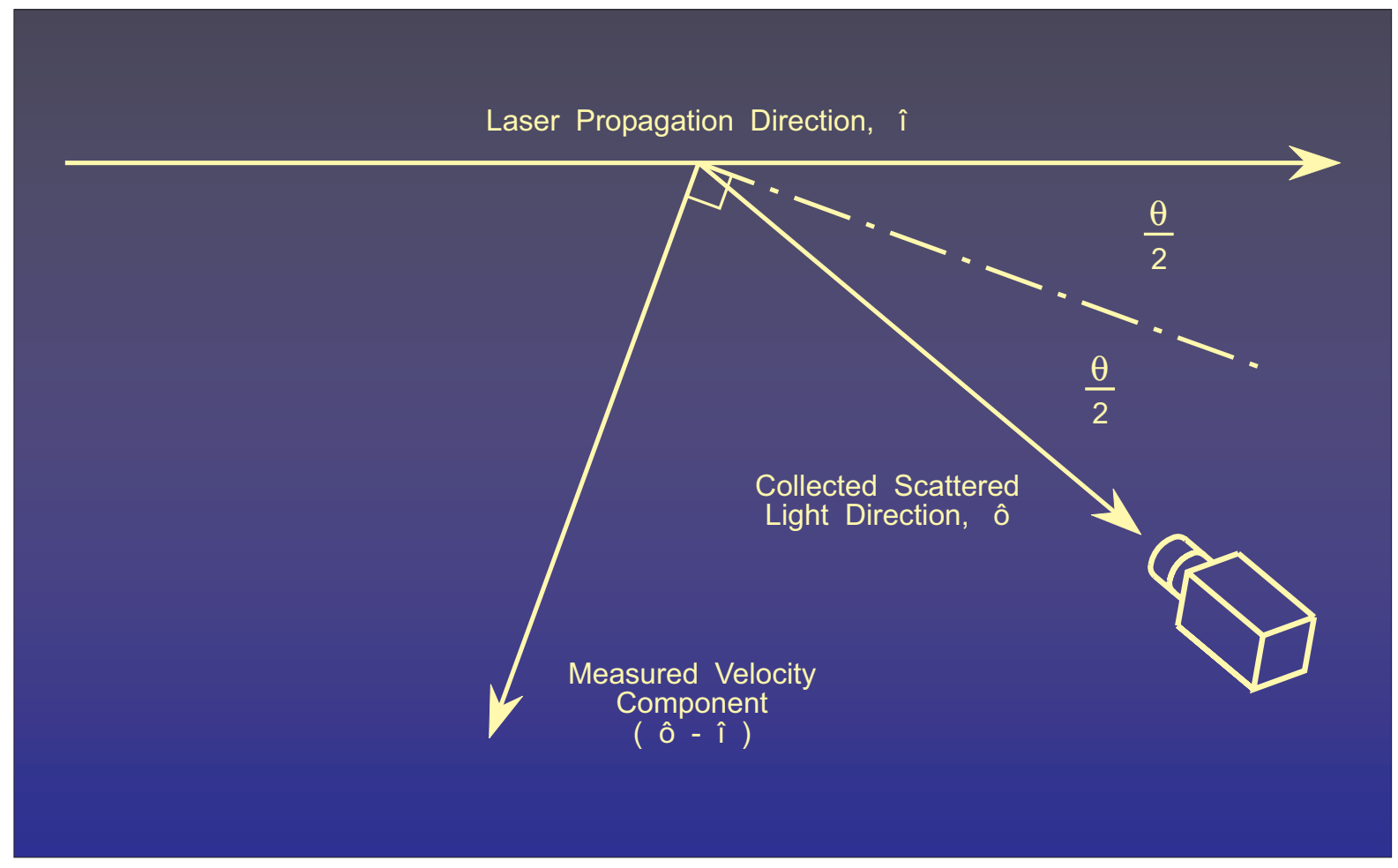

Figure 1. Diagram depicting the velocity measurement direction based on the orientation of the laser propagation direction and the detector location. 


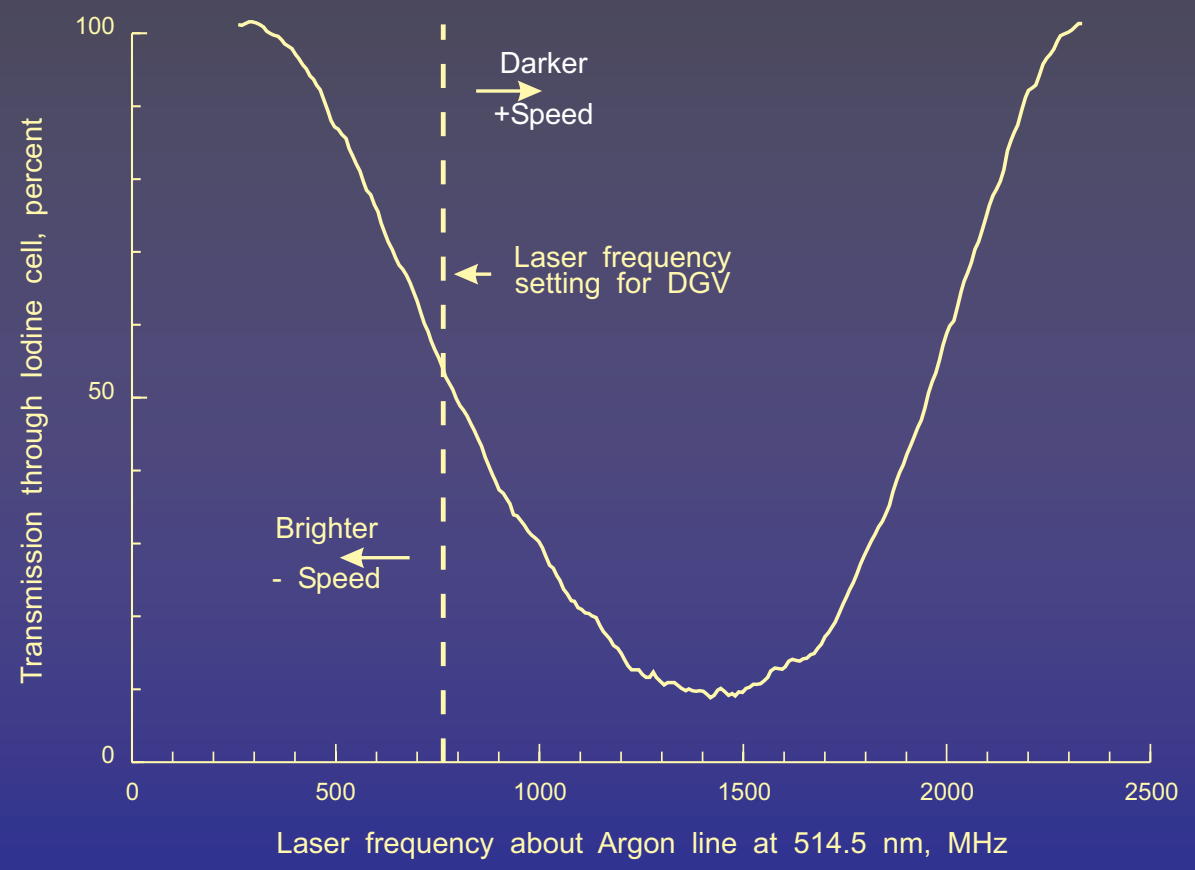

Figure 2. Transfer function of the Iodine vapor ce1l, IVC.

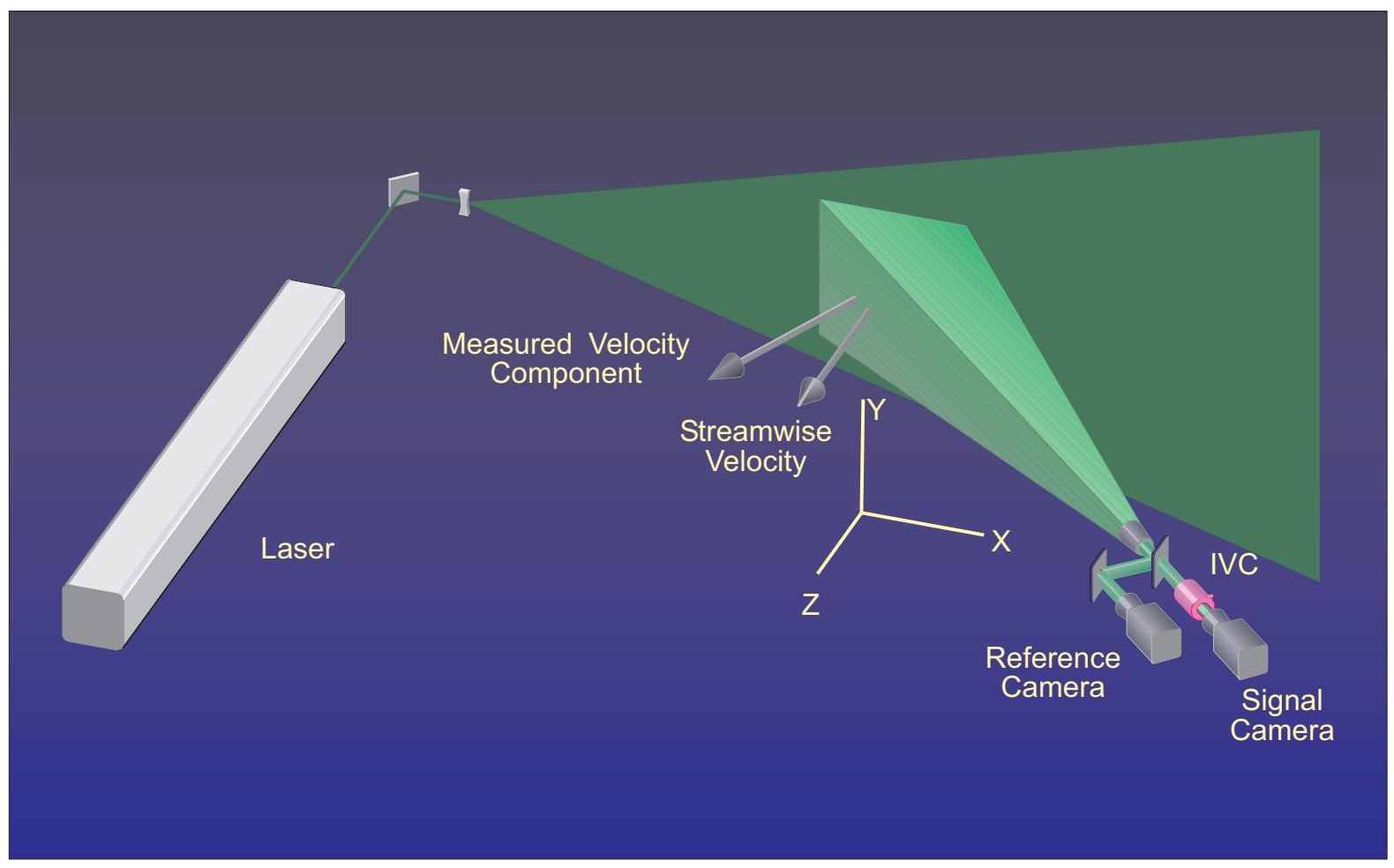

Figure 3. Pictorial view of the Doppler global velocimeter used in the Basic Aerodynamics Research Tunnel to measure the flow above a 75 -degree delta wing. 


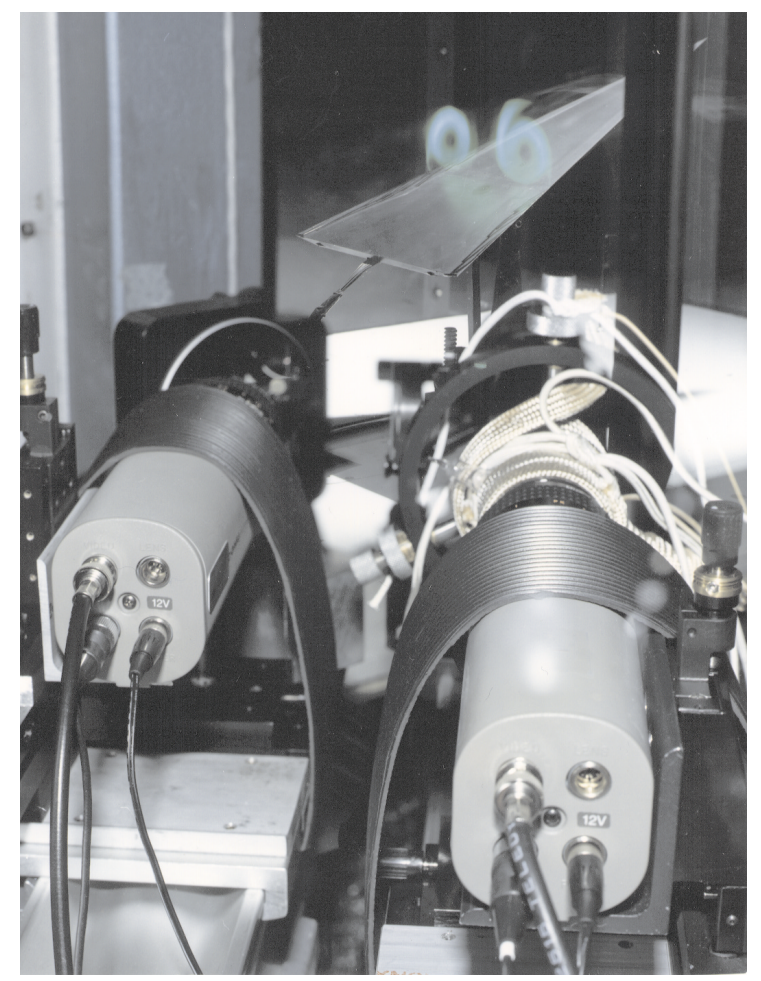

Figure 4. Photograph of the Doppler global velocimeter installed in the Basic Aerodynamics Research Tunnel.

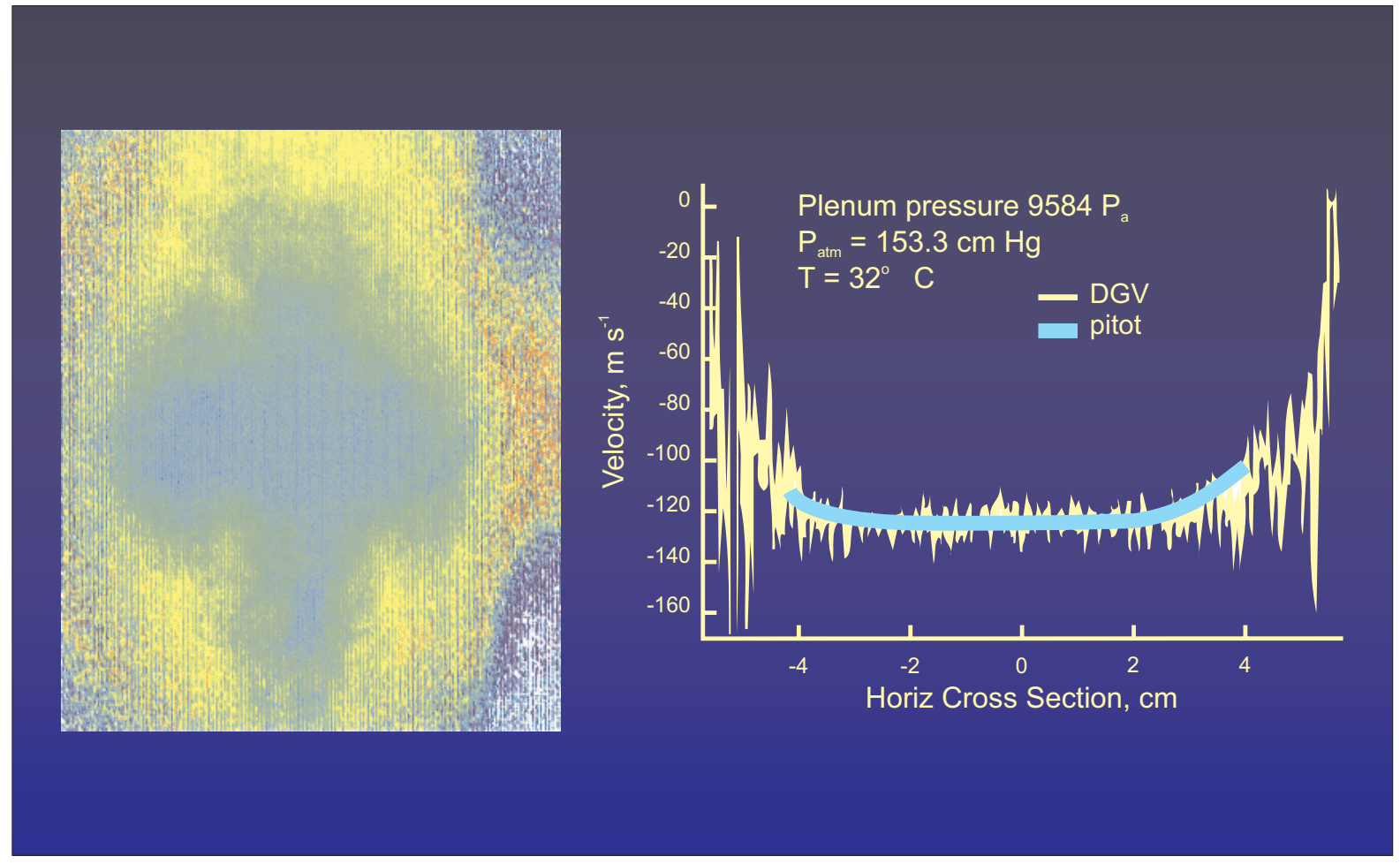

Figure 5. Comparison of velocities obtained with a DGV using a pulsed, frequency-doubled Nd:YAG laser and a pitot tube in a small subsonic jet. (Northrop Corporation - NASA contract NAS1-19940) 


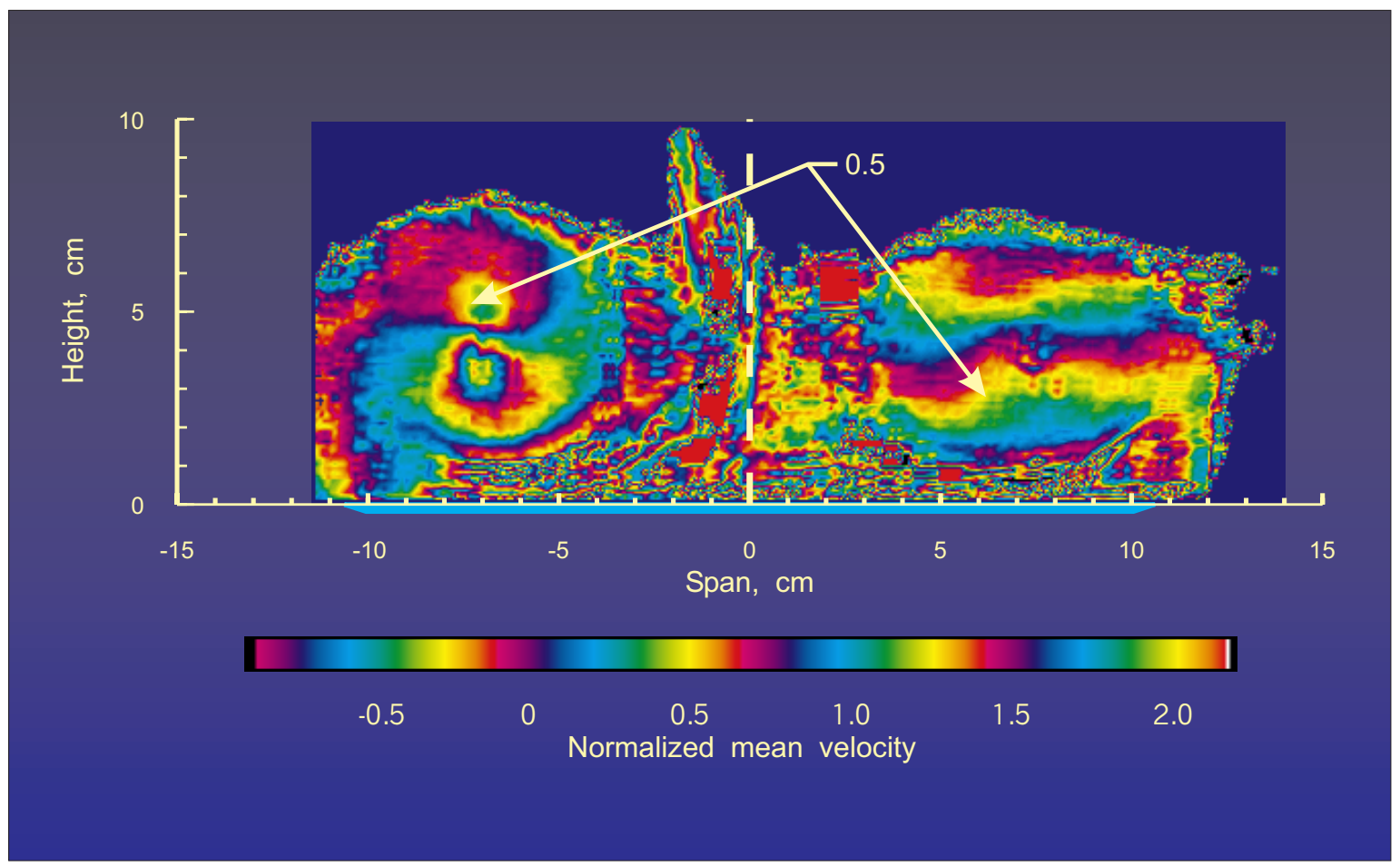

Figure 6. DGV measurements of the velocity field above a 75-degree delta wing at an angle of attack of 20.5 degrees, for the component along the direction 71.5 degrees from streamwise in the horizontal plane.

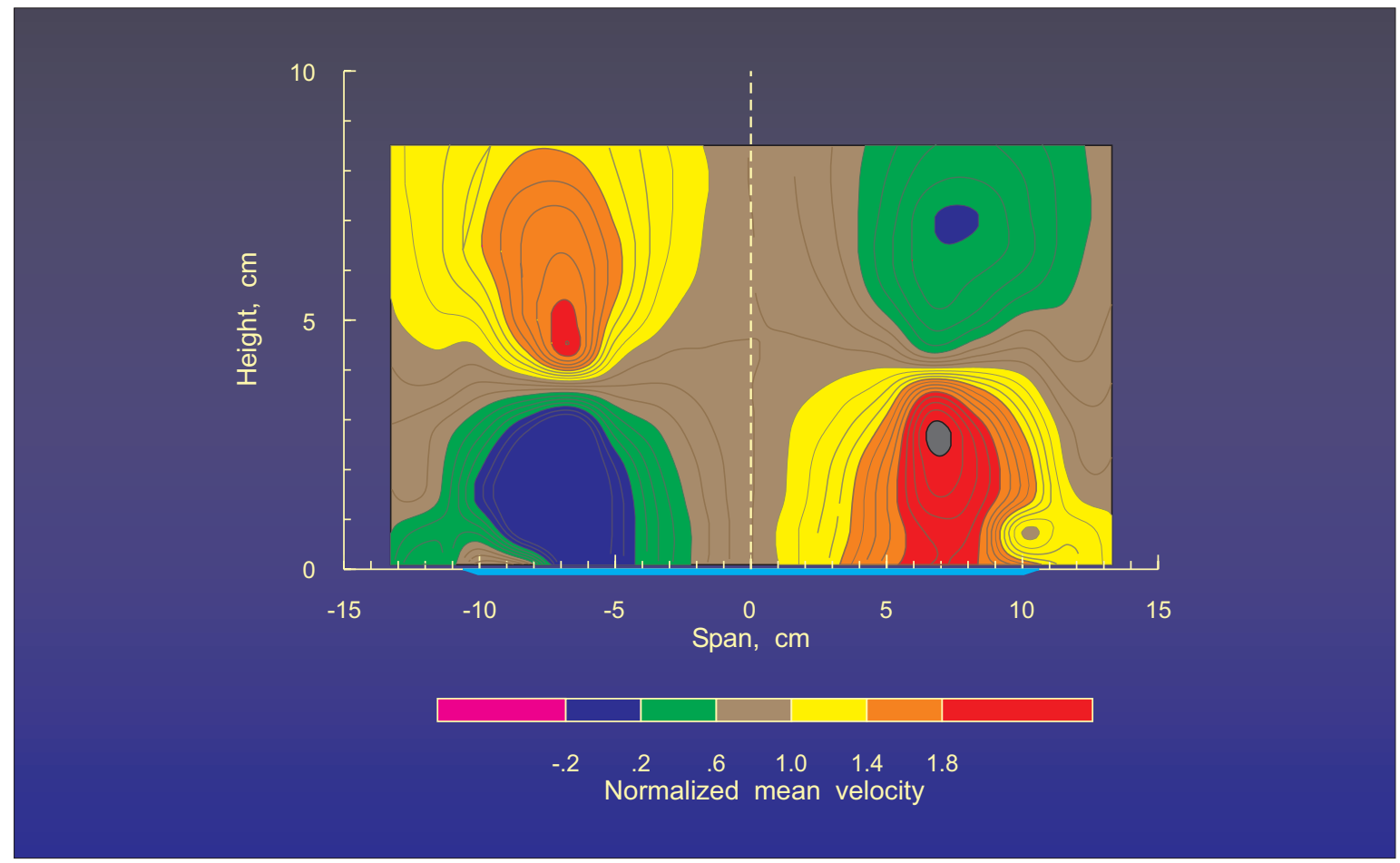

Figure 7. Laser velocimetry measurements of the velocity field above a 75-degree delta wing at an angle of attack of 20.5 degrees, resolved along the same velocity vector as the DGV measurements in Figure 6. 


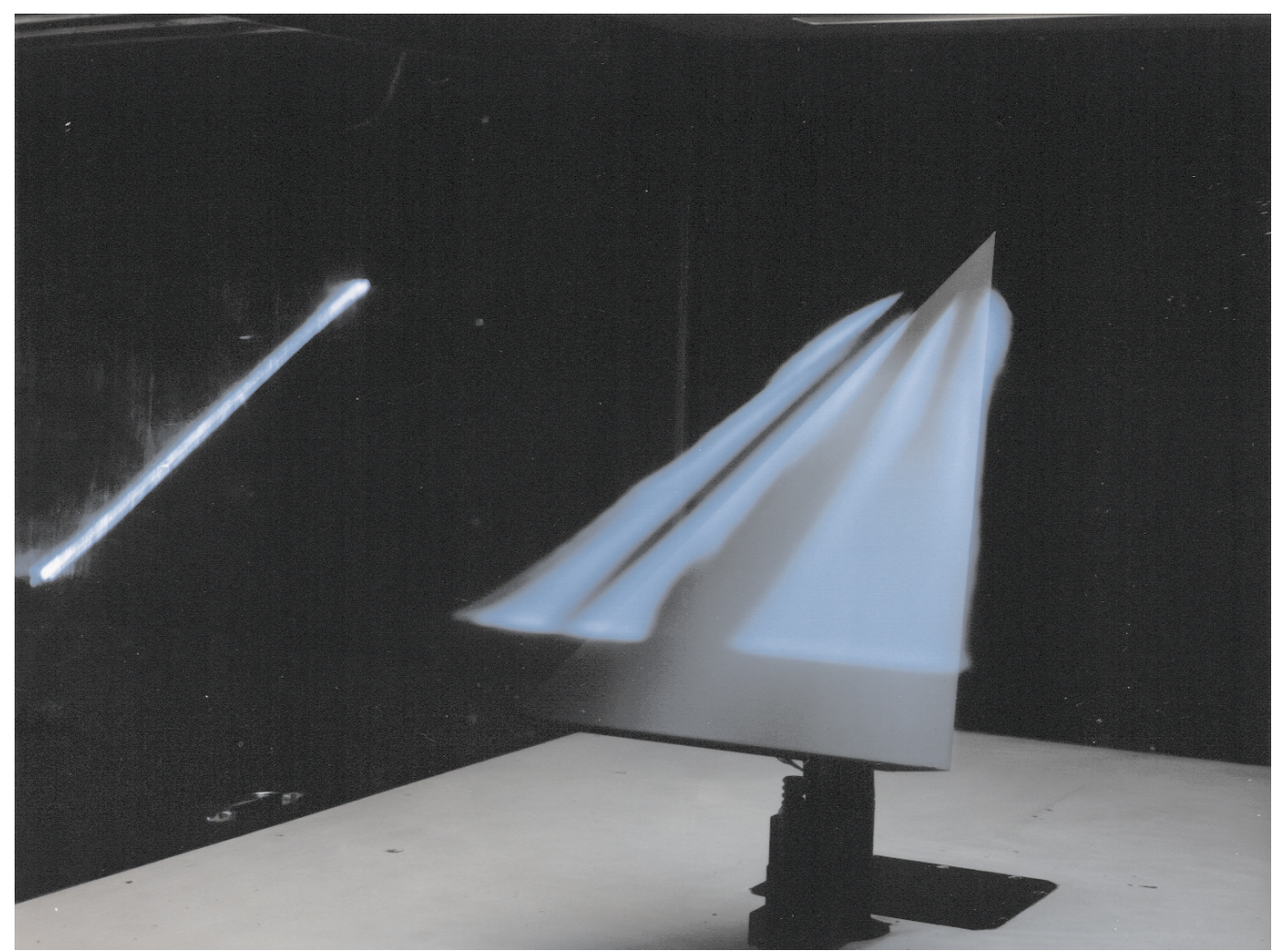

Figure 8. Flow visualization of the vortical flow above a 75 -degree delta wing at an angle of attack of 20.5 degrees.

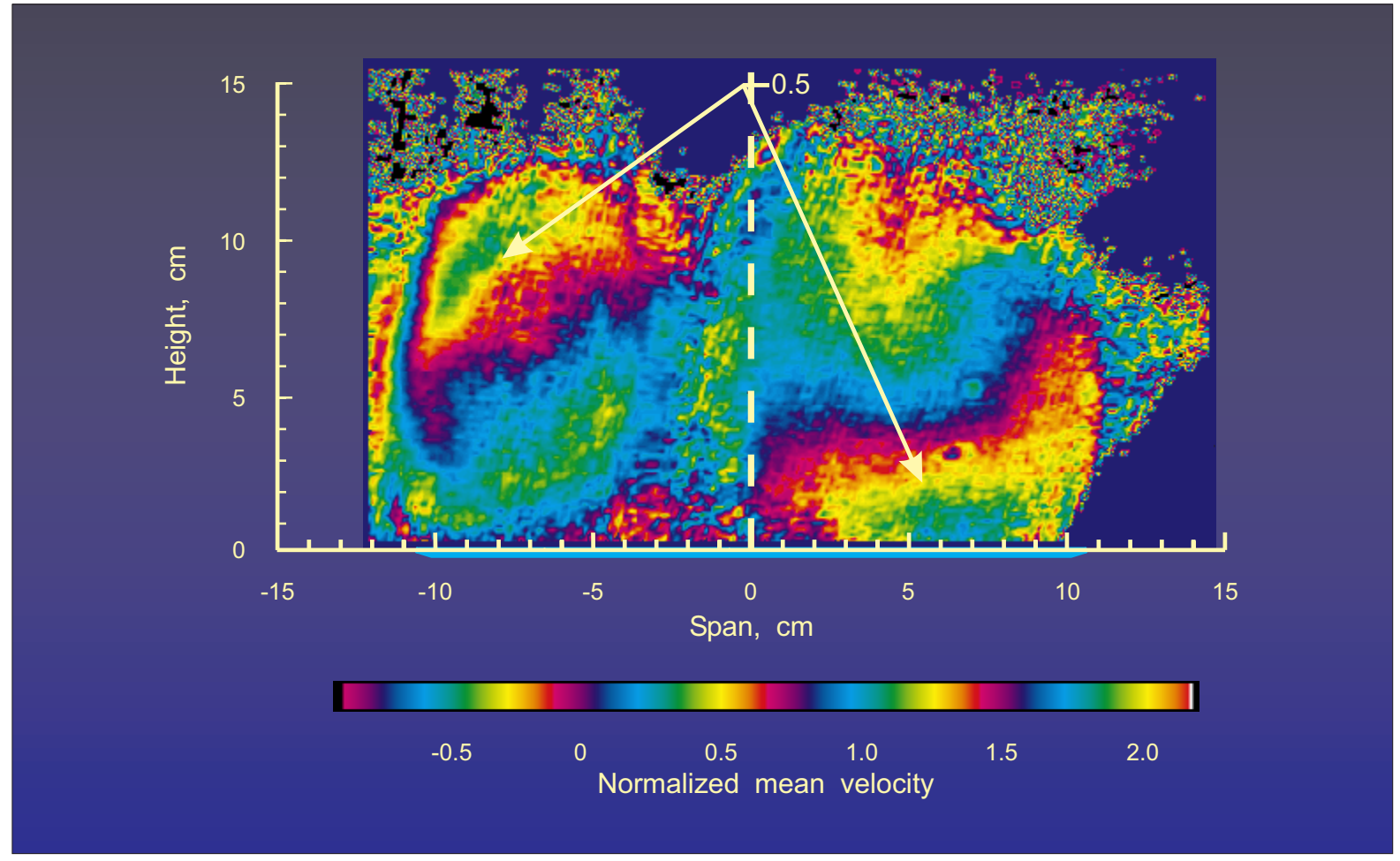

Figure 9. DGV measurements of the velocity field above a 75-degree delta wing at an angle of attack of 40.0 degrees, for the component along the direction 71.5 degrees from streamwise in the horizontal plane. 


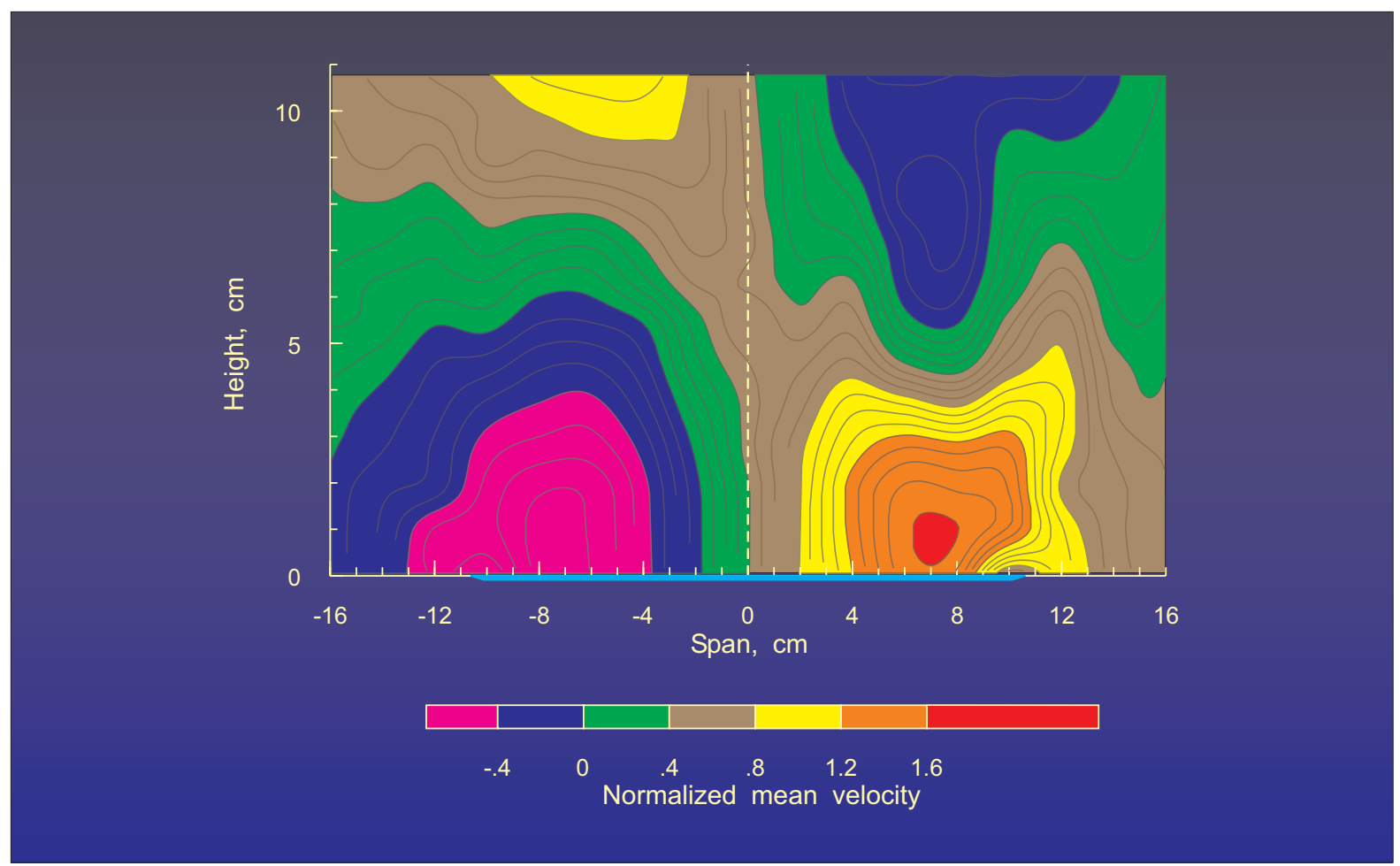

Figure 10. Laser velocimetry measurements of the velocity field above a 75-degree delta wing at an angle of attack of 40.0 degrees, resolved along the same velocity vector as the DGV measurements in Figure 9.

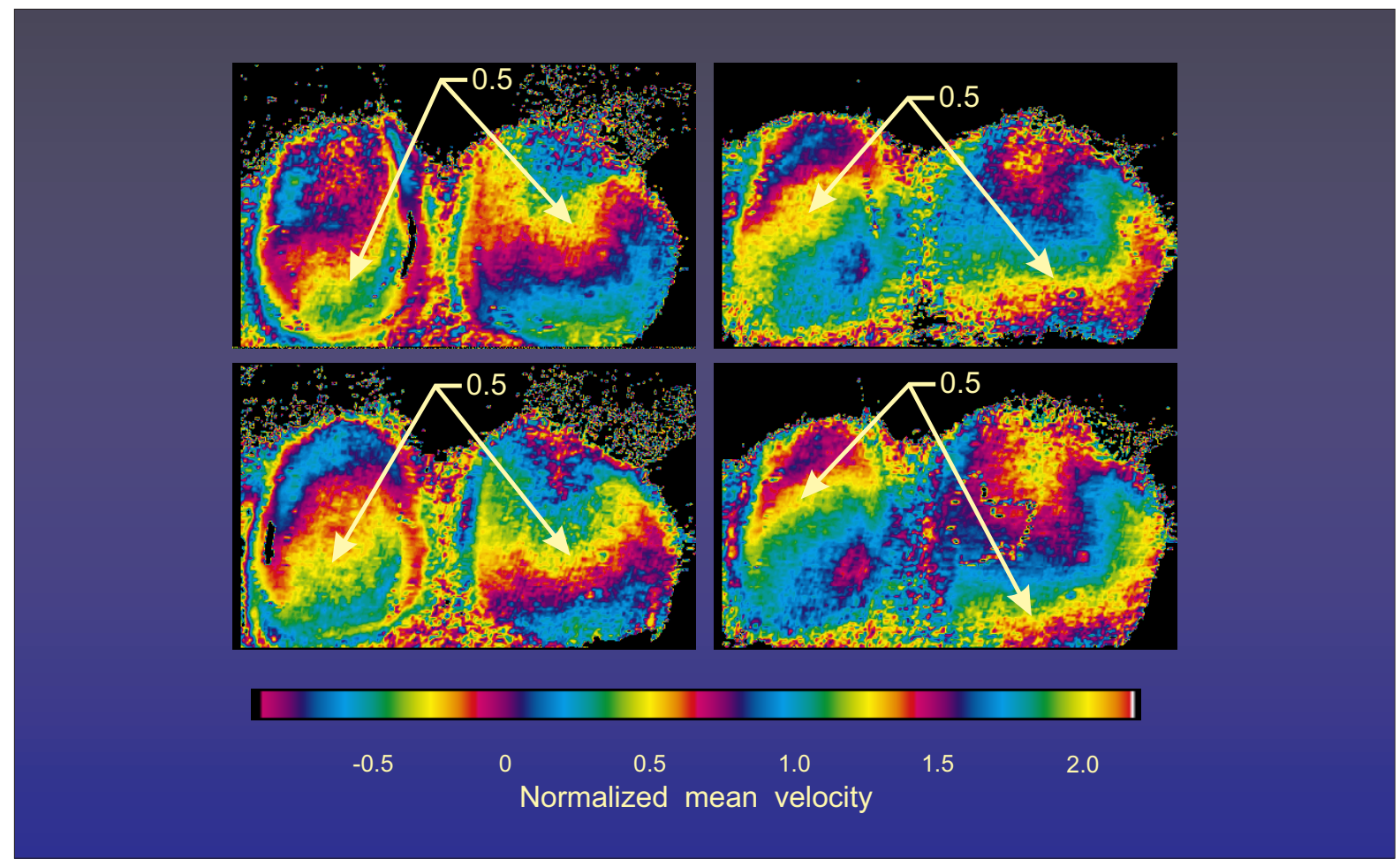

Figure 11. Individual field images comprising a portion of the data averaged in Figure 9. 


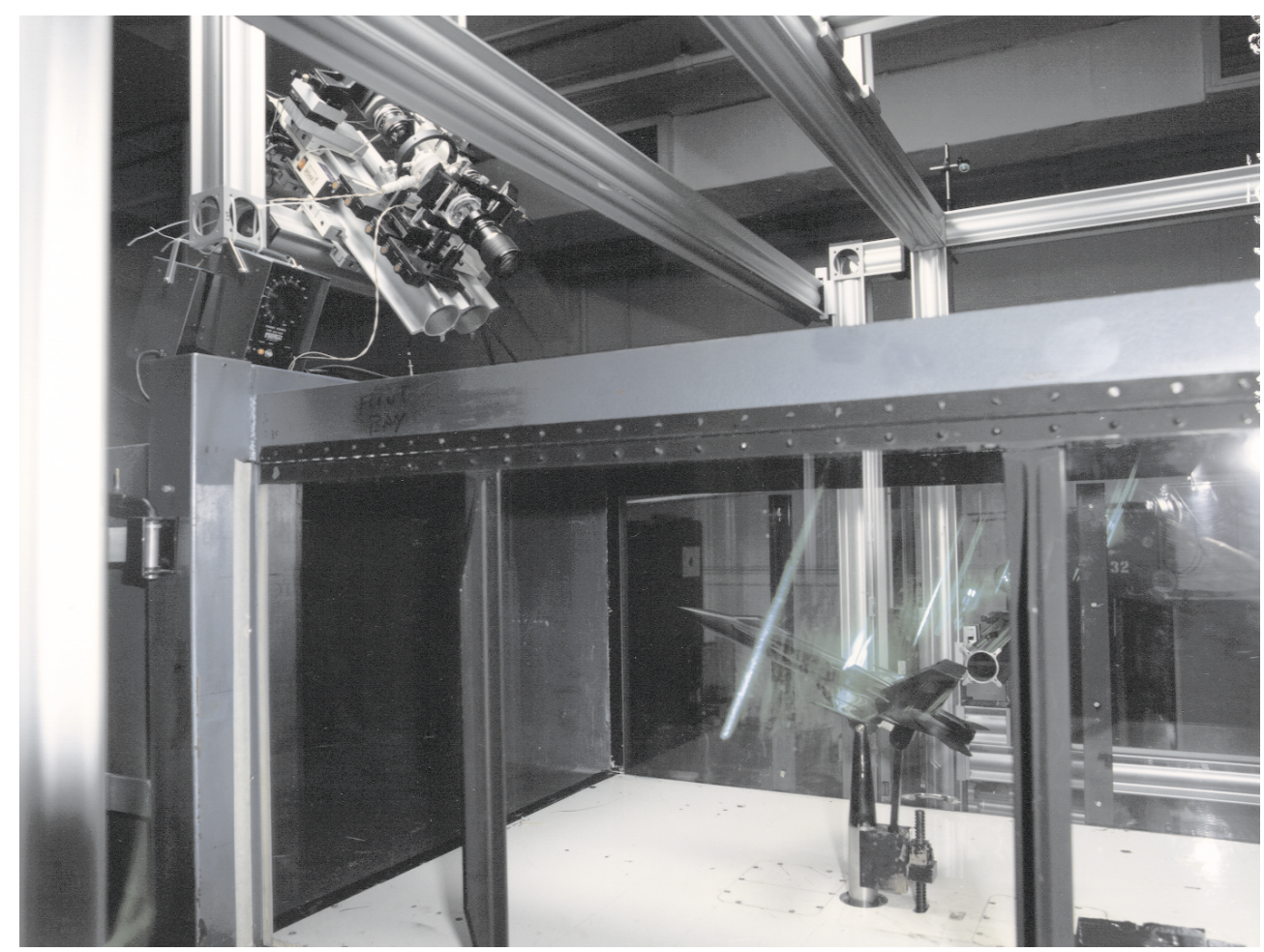

Figure 12. Pictorial view of the Doppler global velocimeter used in the Basic Aerodynamics Research Tunnel to measure the flow above an F/A-18 model.

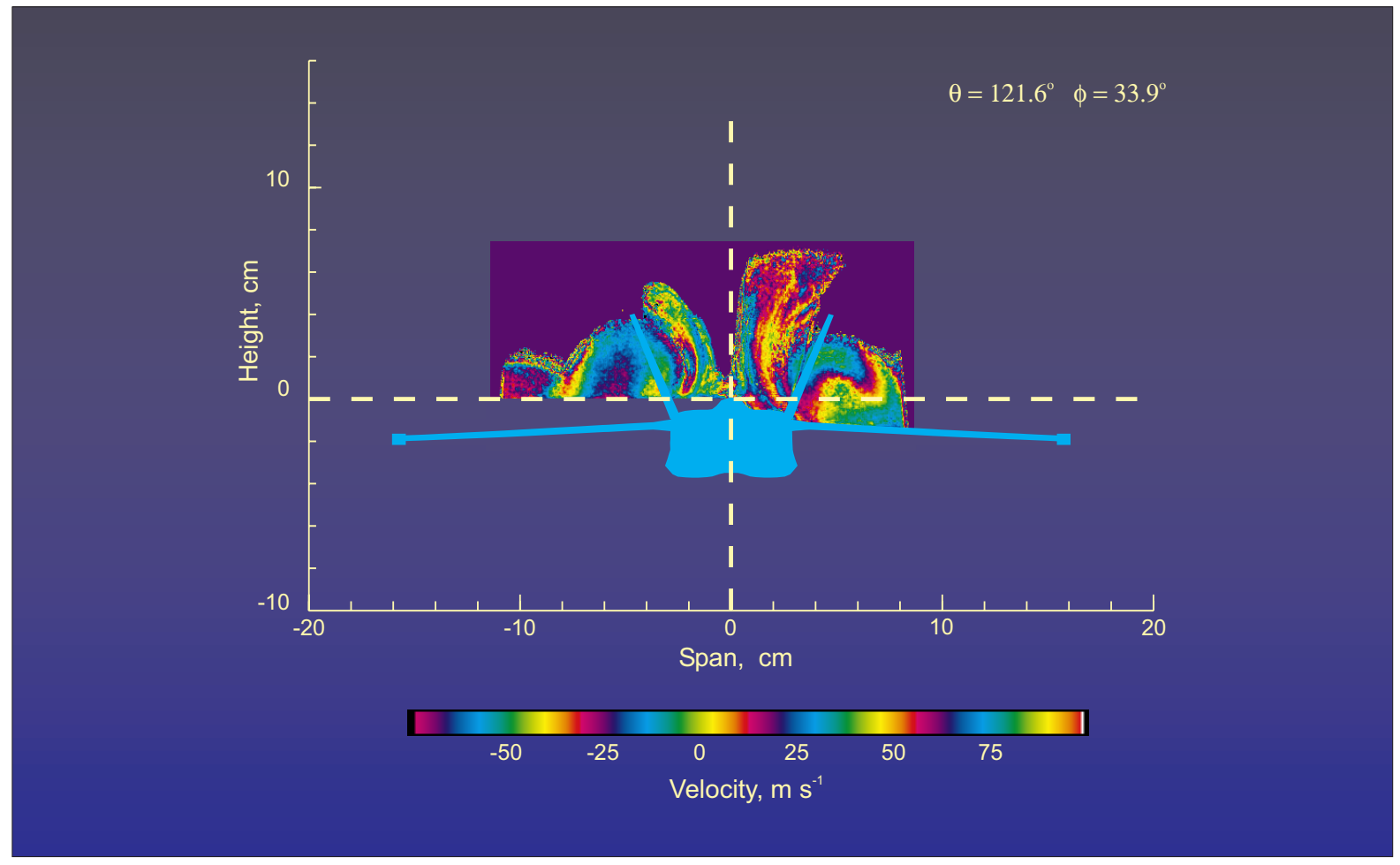

Figure 13. DGV measurements of the velocity field above an F/A-18 model at an angle of attack of 25 degrees for the component along the direction 53.0 degrees from streamwise and 45.0 degrees above the horizontal plane. 


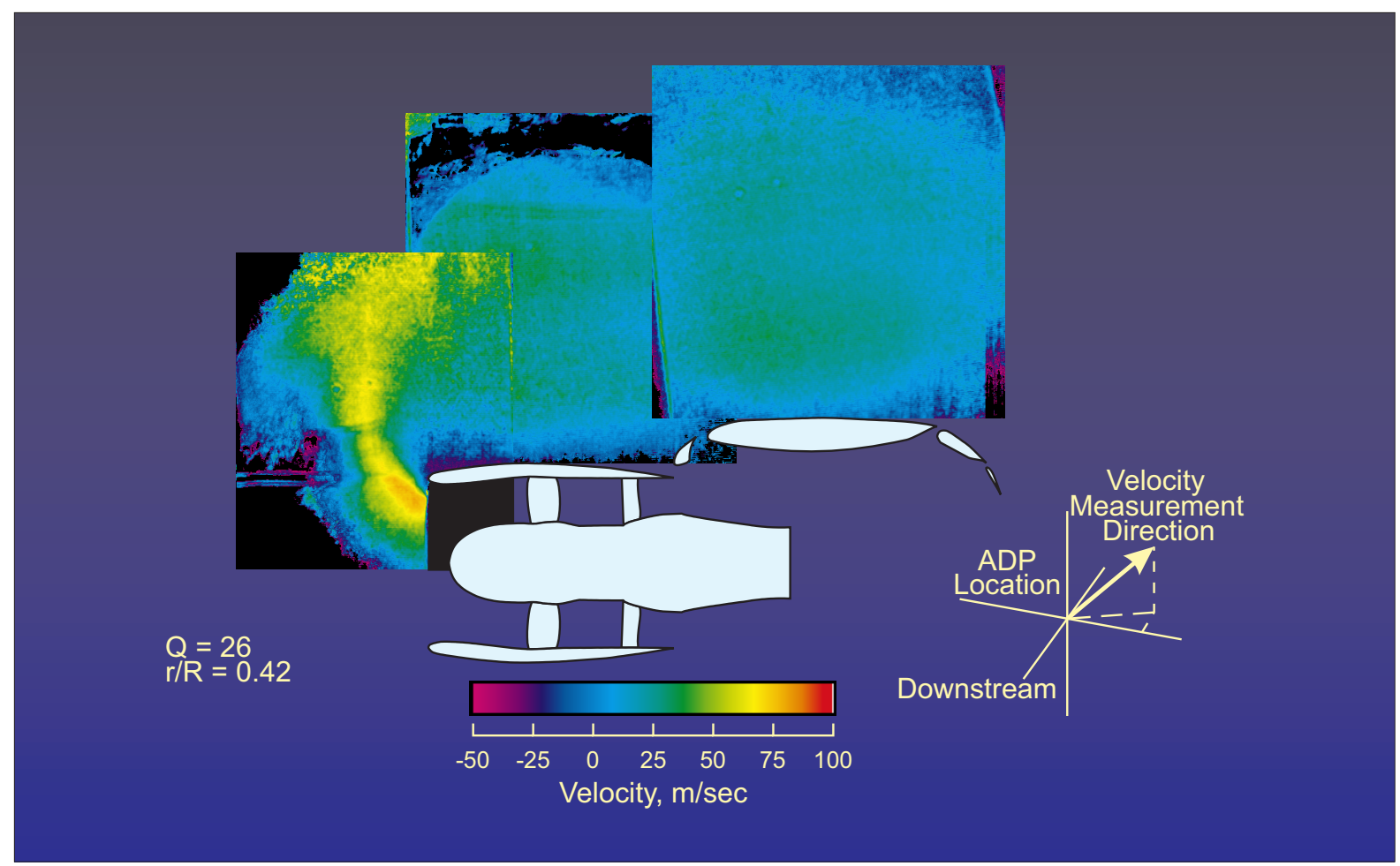

Figure 14. DGV measurements of jet flow interactions with free stream from an engine operating in reverse thrust.

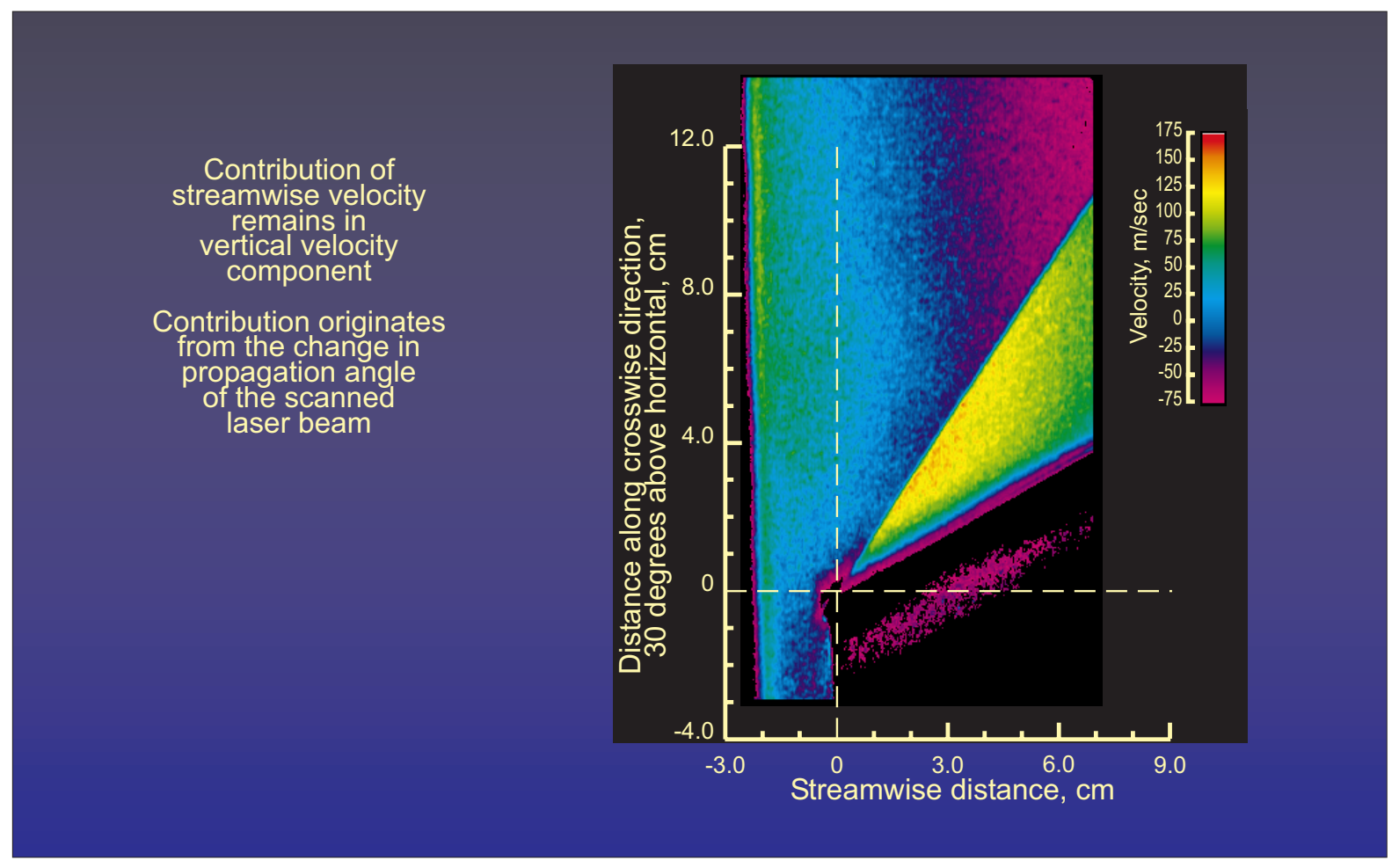

Figure 15. Map of the vertical velocity component measured by the DGV of the flow above a flat plate inclined to -15 degrees at Mach 2.5. Contributions of the free stream flow are included in the data. 


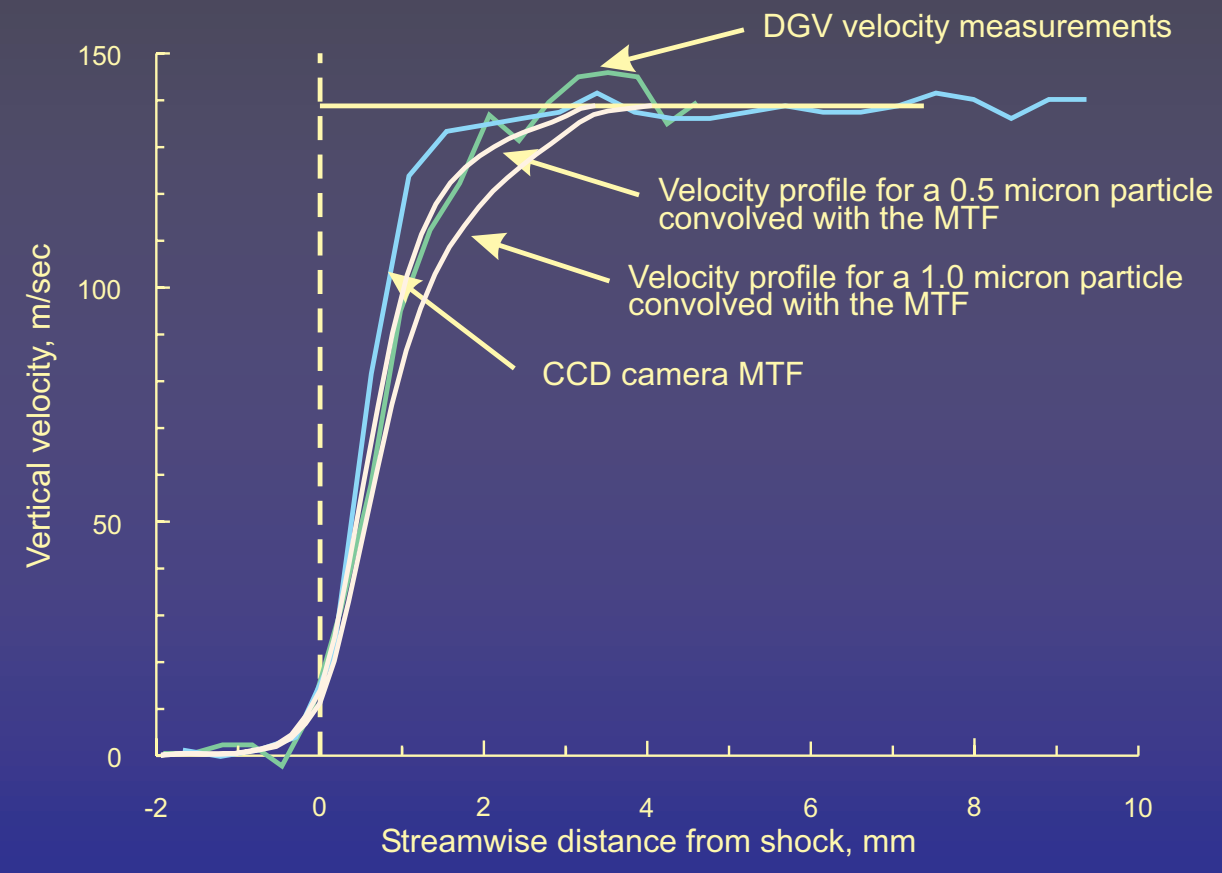

Figure 16. Comparison of the DGV measurements across the shock with theoretical responses for $0.5 \mathrm{micron}$ and $1.0 \mathrm{micron}$ particles. The CCD camera modulation transfer function indicates the limits of the instrument.

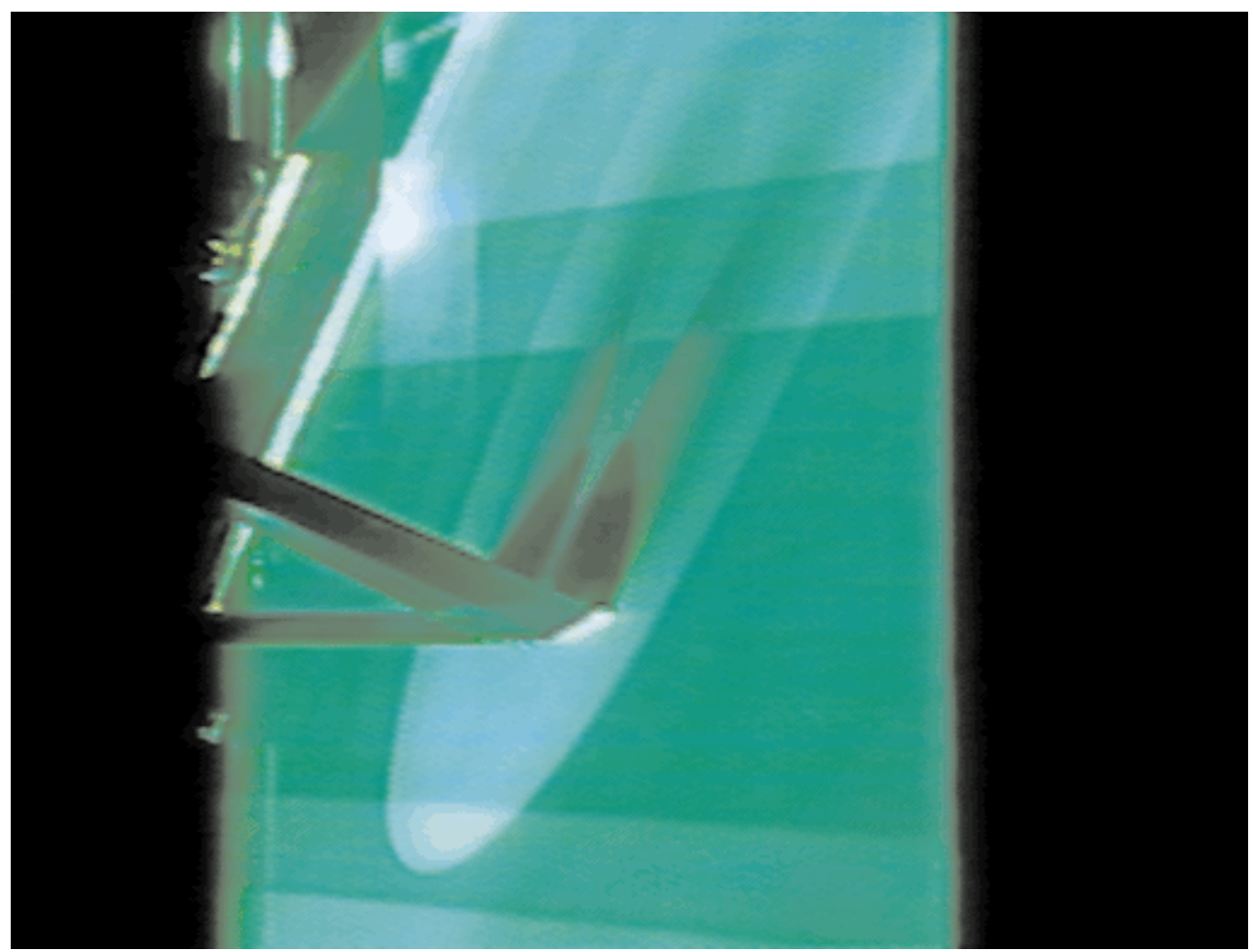

Figure 17. Photograph of the laser light sheet above a 75-degree delta wing at the 95-percent chord location at Mach 2.8. 


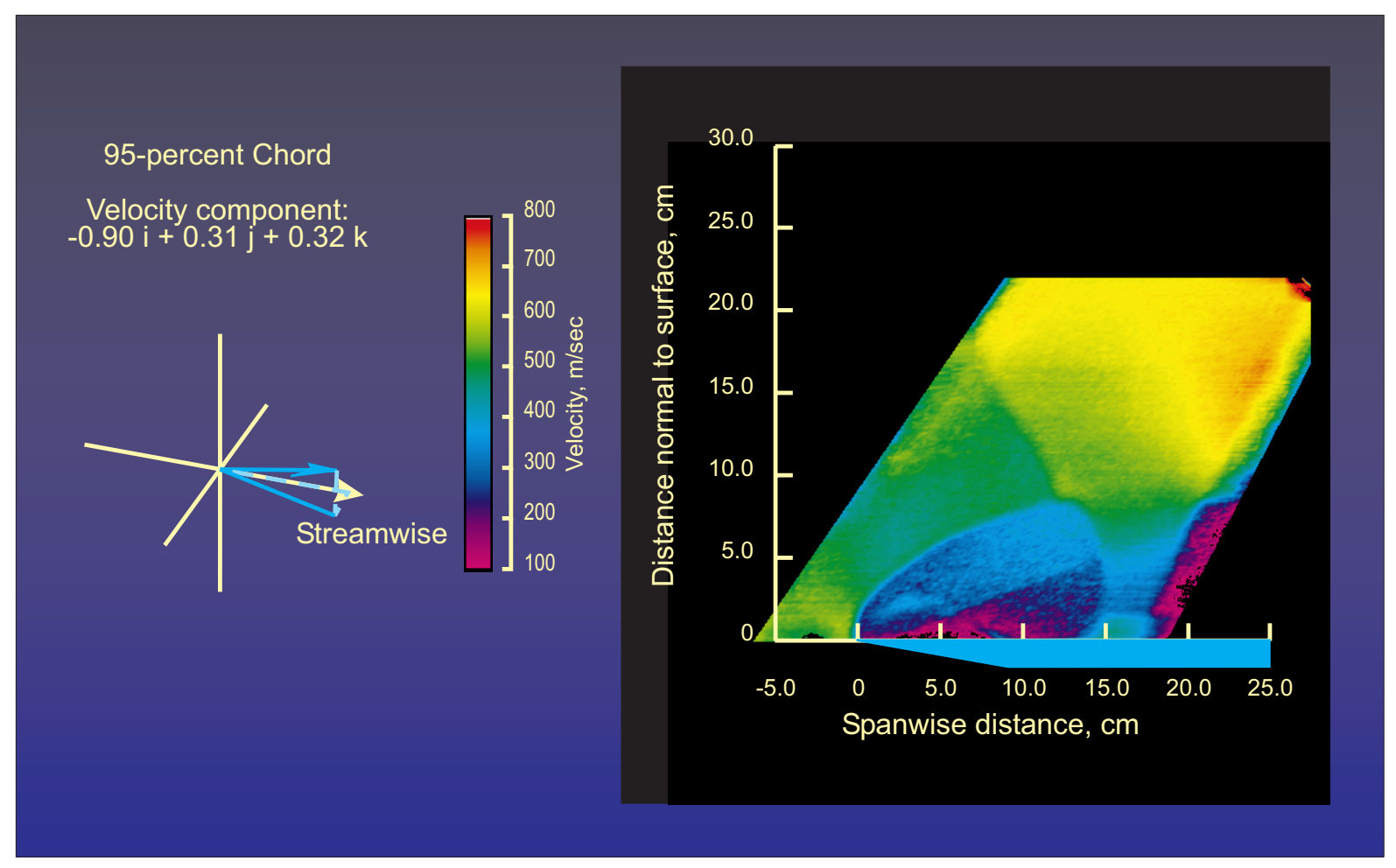

Figure 18. Map of the velocity flow measured by the DGV of the vortical flow above a 75-degree delta wing at the 95-percent chord location at Mach 2.8.

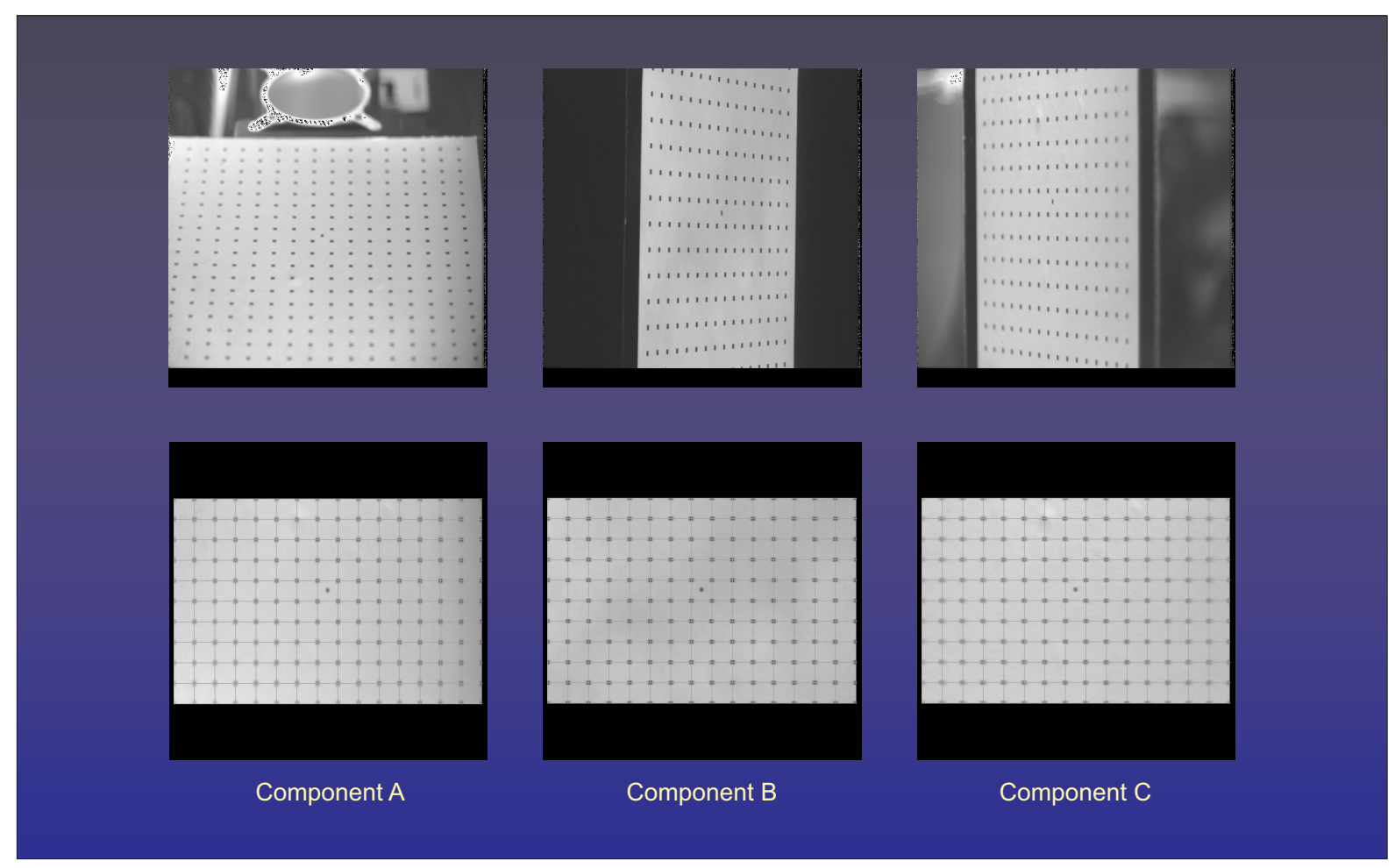

Figure 19. Views of equally spaced dots on a flat card from the 1eft, right, and above with a inclination of 30 degrees from the card plane, before and after warping. 


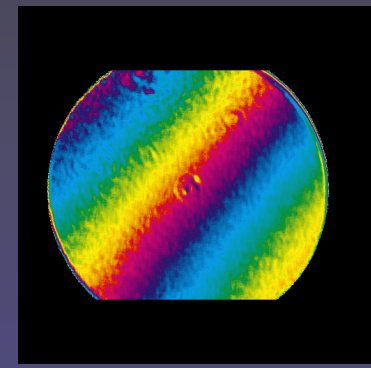

A

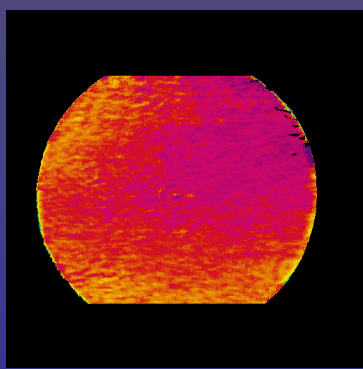

U

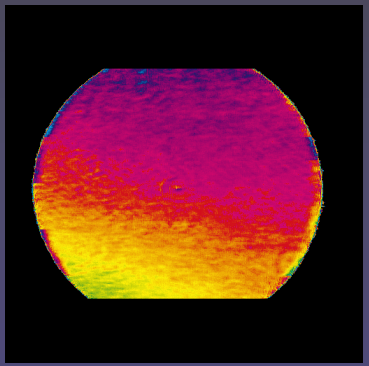

B

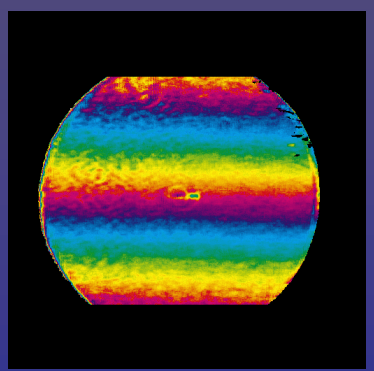

V

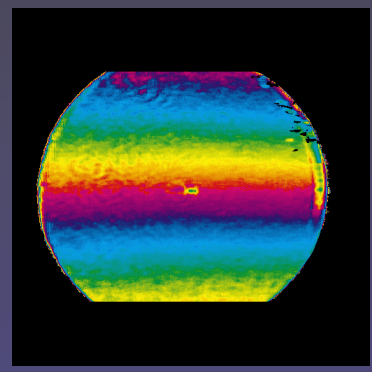

C

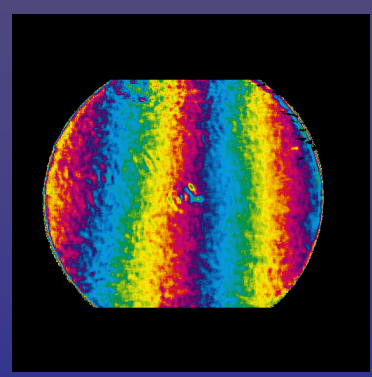

W

Figure 20. Original and resolved $u, v$, and $w$ velocity component images of a rotating wheel obtained from the three views shown in figure 19.

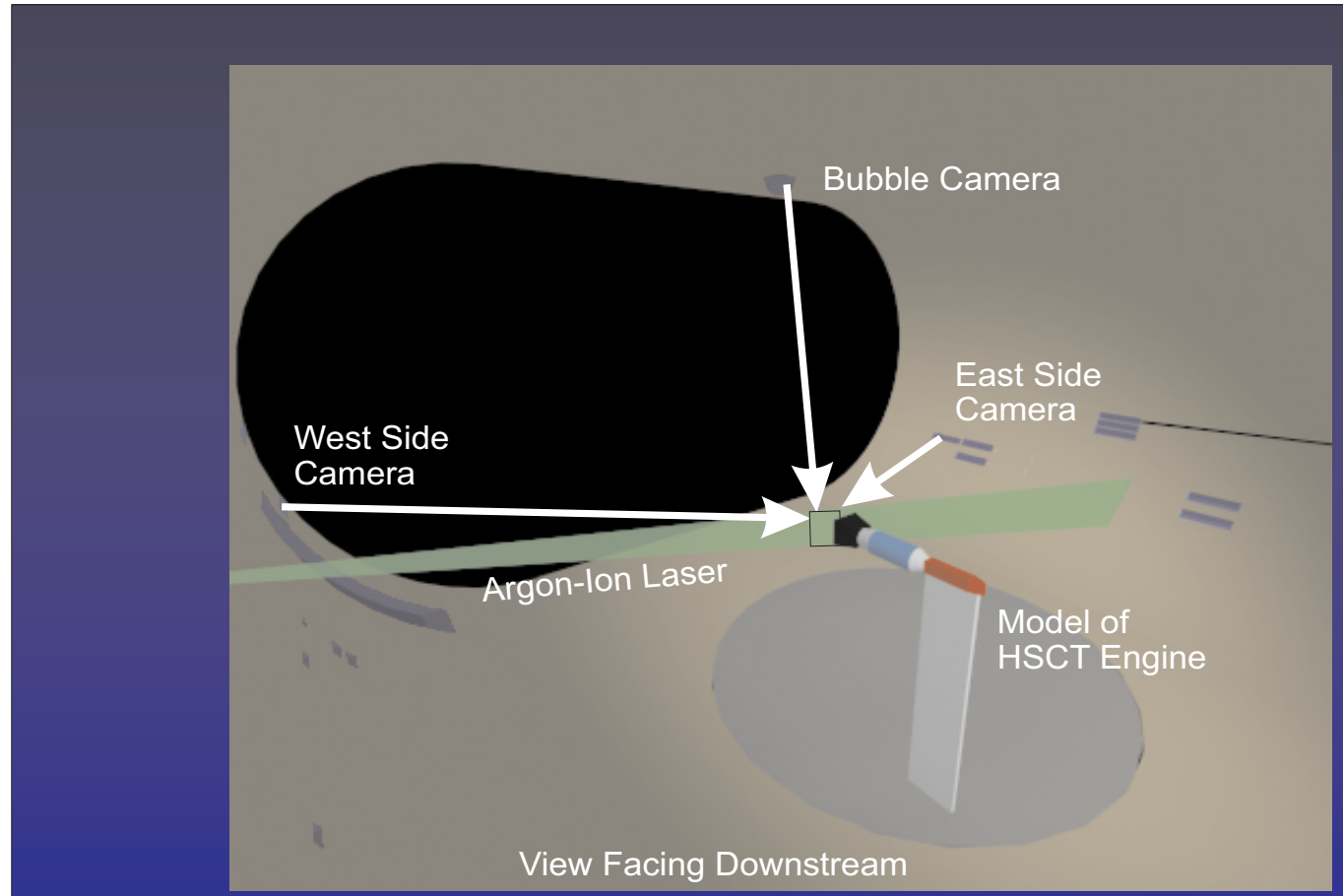

Figure 21. Configuration of the three-component DGV optical system in the NASA Ames Research Center 40-x80-foot Wind Tunnel to measure the flow from a high-speed jet. 


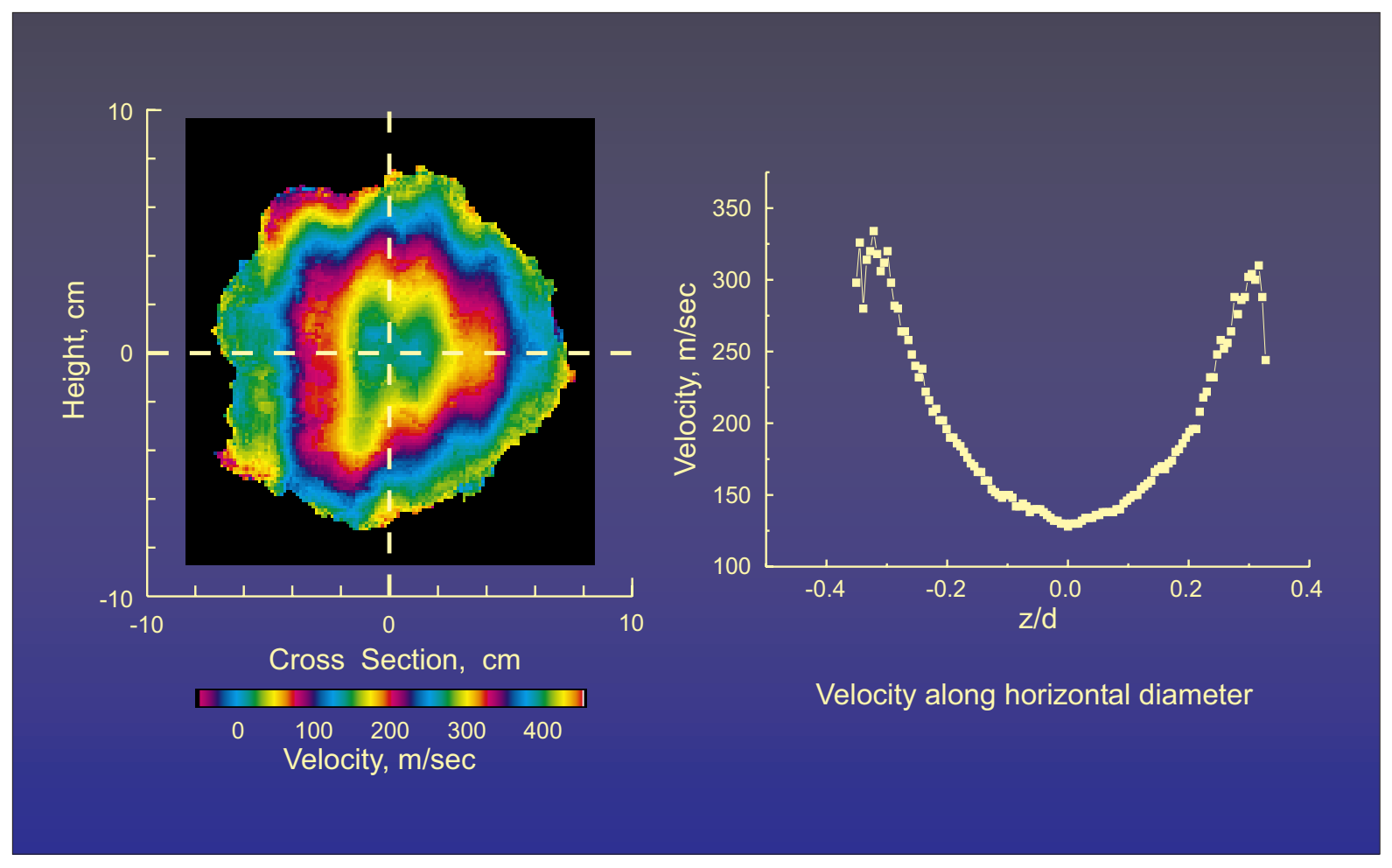

Figure 22. Resolved streamwise component of velocity from the high-speed jet flow operating at $463^{\circ} \mathrm{C}$ at a free stream Mach number equal to 0.15 . 